\title{
Proton irradiated graphite grades for a long baseline neutrino facility experiment
}

\author{
N. Simos, ${ }^{1,}{ }^{*}$ P. Nocera, ${ }^{3}$ Z. Zhong, ${ }^{1}$ R. Zwaska, ${ }^{2}$ N. Mokhov, ${ }^{2}$ J. Misek, ${ }^{2}$ \\ K. Ammigan, ${ }^{2}$ P. Hurh, ${ }^{2}$ and Z. Kotsina ${ }^{4}$ \\ ${ }^{1}$ Brookhaven National Laboratory, Upton, New York 11973, USA \\ ${ }^{2}$ Fermi National Accelerator Laboratory, Batavia, Illinois 60510-5011, USA \\ ${ }^{3}$ Department of Physics, University of Rome, 20122 Rome, Italy \\ ${ }^{4}$ Department of Solid State Physics, National and Kapodistrian University of Athens, \\ University Campus, GR-157 84 Athens, Greece \\ (Received 9 April 2016; published 24 July 2017)
}

\begin{abstract}
In search of a low-Z pion production target for the Long Baseline Neutrino Facility (LBNF) of the Deep Underground Neutrino Experiment (DUNE) four graphite grades were irradiated with protons in the energy range of $140-180 \mathrm{MeV}$, to peak fluence of $\sim 6.1 \times 10^{20} \mathrm{p} / \mathrm{cm}^{2}$ and irradiation temperatures between $120-200{ }^{\circ} \mathrm{C}$. The test array included POCO ZXF-5Q, Toyo-Tanso IG 430, Carbone-Lorraine 2020 and SGL R7650 grades of graphite. Irradiation was performed at the Brookhaven Linear Isotope Producer. Postirradiation analyses were performed with the objective of (a) comparing their response under the postulated irradiation conditions to guide a graphite grade selection for use as a pion target and (b) understanding changes in physical and mechanical properties as well as microstructure that occurred as a result of the achieved fluence and in particular at this low-temperature regime where pion graphite targets are expected to operate. A further goal of the postirradiation evaluation was to establish a proton-neutron correlation damage on graphite that will allow for the use of a wealth of available neutron-based damage data in proton-based studies and applications. Macroscopic postirradiation analyses as well as energy dispersive $\mathrm{x}$-ray diffraction of $200 \mathrm{KeV} \mathrm{x}$ rays at the NSLS synchrotron of Brookhaven National Laboratory were employed. The macroscopic analyses revealed differences in the physical and strength properties of the four grades with behavior however under proton irradiation that qualitatively agrees with that reported for graphite under neutrons for the same low temperature regime and in particular the increase of thermal expansion, strength and Young's modulus. The proton fluence level of $\sim 10^{20} \mathrm{~cm}^{-2}$ where strength reaches a maximum before it begins to decrease at higher fluences has been identified and it agrees with neutron-induced changes. X-ray diffraction analyses of the proton irradiated graphite revealed for the first time the similarity in microstructural graphite behavior to that under neutron irradiation and the agreement between the fluence threshold of $\sim 5 \times 10^{20} \mathrm{~cm}^{-2}$ where the graphite lattice undergoes a dramatic change. The confirmed similarity in behavior and agreement in threshold fluences for proton and neutron irradiation effects on graphite reported for the first time in this study will enable the safe utilization of the wealth of neutron irradiation data on graphite that extends to much higher fluences and different temperature regimes by the proton accelerator community searching for multi-MW graphite targets.
\end{abstract}

DOI: 10.1103/PhysRevAccelBeams.20.071002

\section{INTRODUCTION}

In search of a low-Z pion production target for the Long Baseline Neutrino Facility (LBNF) of the Deep Underground Neutrino Experiment (DUNE) four graphite grades were irradiated with protons in the energy range of $140-180 \mathrm{MeV}$, to peak fluence of $\sim 6.1 \times 10^{20} \mathrm{p} / \mathrm{cm}^{2}$ and irradiation temperatures between $120-200^{\circ} \mathrm{C}$ [1]. In next

\footnotetext{
*Corresponding author. simos@bnl.gov

Published by the American Physical Society under the terms of the Creative Commons Attribution 3.0 License. Further distribution of this work must maintain attribution to the author(s) and the published article's title, journal citation, and DOI.
}

generation multi-MW power accelerators such as the LBNF/ DUNE high-performance and high reliability particle production targets are required. The LBNF plans for a design of a 2+ MW, 60-120 GeV pulsed, high intensity proton beam produced in the accelerator to be intercepted by a low-Z solid target and facilitate the production of low energy neutrinos. The multi-MW level LBNF proton beam will be characterized by intensities of $\sim 1.6 \times 10^{14}$ protons/pulse, $\sigma$ of $1.5-3.5 \mathrm{~mm}$ and a $9.8 \mu$ s pulse length. These parameters are expected to push several choice target materials, desirable for their physics performance, to their limit and beyond thus making the design and operation of a target system extremely challenging. Graphite has been the target material of choice for several neutrino beam experiments to date such as NuMI/MINOS, T2K, CNGS because of its low-Z, good 
resistance to thermal shock, one of the key concerns in designing high-power targets, and its track record in surviving high neutron fluences in fission reactors. Because of its lattice structure graphite undergoes changes in important physical properties under irradiation at relatively low doses and in particular at low temperatures and thus this regime of dose-temperature warrants special attention. Correlation between proton-induced effects with those caused by neutrons is of paramount importance towards establishing operational limits due to the availability of a wealth of neutron-related data on graphite from decades of research work for nuclear reactor operations. Neutron data on graphite extend to much higher fluences as well as irradiation temperature regimes that, following a successful correlation with protons, can be utilized in establishing an optimal operating envelope for multi-MW accelerator targets such as LBNF.

The four irradiated graphite grades were POCO ZXF-5Q (POCO), Toyo-Tanso IG 430 (IG 430), Carbone-Lorraine 2020 (C-2020) and SGL R7650 (SGL). Irradiation was performed at the Brookhaven Linear Isotope Producer (BLIP). Macroscopic and microscopic postirradiation analyses were performed on these four graphite grades with the objective of (a) comparing their response under the postulated irradiation conditions to guide a graphite grade selection for use as a pion target and (b) understanding changes in physical and mechanical properties as well as microstructure that occurred as a result of the achieved fluence and in particular at this low-temperature regime where pion graphite targets are expected to operate. Proton irradiation effects of the four graphite grades were evaluated by focusing on dimensional stability and changes in thermal expansion coefficient, thermal annealing, irradiation-induced changes in mechanical strength and Young's modulus and their postirradiation recovery through annealing. Changes in the microstructure were evaluated using energy dispersive $\mathrm{x}$-ray diffraction (EDXRD) and the $200 \mathrm{KeV} x$ rays at the NSLS synchrotron of Brookhaven National Laboratory (BNL).

A large body of work on irradiated graphite by reactor neutrons (primarily thermal and to a lesser extent fast) exists [2-8]. Extrapolation, however, of the performance of graphite on the basis of accumulated fluence alone is risky and therefore direct comparison and use of irradiation damage findings and physiomechanical property evolution should always be made with extreme caution. Irradiation induced deformations of graphite and carbon, including irradiation creep and postirradiation annealing are discussed in [3] where a number of works on irradiated graphite are used as moderators in reactors. Specifically, the effects of high neutron doses in reducing porosity introduced during manufacturing by graphite crystal growth resulting in the "tightening" of the aggregate structure, the rise in the elastic modulus and the partial recovery due to high temperature annealing are discussed on the basis of experience and experimental data. An important point of direct relevance to the present study, also noted in [3], is the observed behavior of isotropic graphite which due to irradiation-induced dimensional changes of individual grains tends to shrink under irradiation. Important work conducted at Brookhaven National Laboratory's research reactors in the 1960s and in particular the establishment of activation energy for annealing interstitials in neutron irradiated graphite is presented in [2]. The rate of growth (volumetric change) as a function of irradiation temperature, an important effect being evaluated in the current study, is presented showing that the rate of growth changes by a factor of 8 from 80 to $220^{\circ} \mathrm{C}$. The percentage of $c$-axis expansion per MWD (MW days, $1 \mathrm{MWD}=7 \times 10^{16} \mathrm{n} \nu \mathrm{t}$ ) of the graphite crystal is listed for fast neutrons and at temperatures similar to the irradiation temperatures of the LBNF graphite grades thus presenting a basis of comparison between damage induced by fast neutrons and energetic protons from the current study.

Bollmann [9] reported on neutron induced damage to graphite including $c$-axis expansion in the graphite crystal as a function of dose as well as stored energy. It is noted in [9] that $c$-axis expansion is linear up to fluence $\sim 6 \times$ $10^{20} \mathrm{n} / \mathrm{cm}^{2}$ beyond which there is broadening in the 002 line. This identified threshold of neutron damage prompted further exploration during recent studies at BNL on protonirradiated graphite to assess whether similar fluence threshold exists for proton damage. Specifically, irradiation damage studies on graphite conducted within the past decade and using $200 \mathrm{MeV}$ protons at the Brookhaven Linear Isotope Producer (BLIP) target station for various accelerator experiments also revealed potential fluence thresholds of energetic protons $\left(\sim 10^{21} \mathrm{p} / \mathrm{cm}^{2}\right)$ which can pose limitations in the use of carbon-based materials (i.e. graphite, $\mathrm{C} / \mathrm{C}$ composites) as targets for long beam exposure. The works of Professor Kelly on irradiation damage in graphite are presented in $[3,10-13]$ where dimensional changes in graphite, including thermal expansion coefficient and fractional changes in Young's modulus as a function of neutron dose, are summarized. Graphite crystal growth in the hexagonal axis and basal plane as a function of neutron dose and irradiation temperature are listed. Experimental data on proton irradiated graphite in the current study will be correlated to the data of [9] in the discussion section.

Graphite lifetime issues are discussed in [14]. Specifically, it is assessed that for "nuclear" graphite, the performance and lifetime not only are closely related to the irradiation environment but also are dramatically affected by the graphite grade choice: manufacturing process, graphitization temperature, etc. It is further reported in [14] that irradiation creep in nuclear graphite occurs at temperatures as low as $100^{\circ} \mathrm{C}$ and can lead to creep deformations 10 times higher than those obtained without irradiation. Nondestructive oxidation damage evaluation of IG 110 and IG 430 graphite 
grades using ultrasonic techniques were studied and reported in [15] indicating that the technique which was also used in the current study but on irradiated graphite is capable of capturing the changes in the elastic modulus.

Prompted by these experimental and operational observations on targets made of materials desired for the multiMW LBNF a comprehensive experimental effort was launched to assess and quantify potential limitations of LBNF target materials using the accelerated damage properties of the BNL $120-200 \mathrm{MeV}$ proton beams. The objectives were to (a) confirm the fluence threshold of the carbon composite when irradiated in a water environment and compare it with the performance of the same target material in an inert gas atmosphere where the potential effects of the water environment are eliminated, (b) evaluate the irradiation-induced changes in the lattice of a various grades of graphite and address damage as a function of proton fluence so estimates of LBNF target life expectancy can be deduced, (c) qualify and quantify the performance of materials/alloys other than graphite and carbon that are within the optimal $\mathrm{Z}$ regime for generating the desired low energy neutrino spectrum and (d) explore potential in situ damage reversal processes that will help extend the LBNF target lifetime. Important in the graphite selection is the confirmation, through the thermal stability studies performed, that isotropic graphite grades such as the highly isotropic POCO and IG 430 undergo microstructural shrinking under irradiation, a behavior that has been previously observed under fast neutron irradiation.
Discussed are details of the proton irradiation experiment, the results of the multifaceted postirradiation evaluation and the assessment of the performance of the graphite grades studied. The primary objective is to assess the applicability and survivability of the selected four graphite grades as high power accelerator pion production targets where the structural integrity, density reduction, dimensional and physical property stability are of paramount importance.

\section{EXPERIMENTAL}

An eight-week irradiation experiment resulting in accumulated flux that is expected to induce damage in the target materials equivalent to one LBNF year of operation at $700 \mathrm{~kW}$ power was undertaken. The test matrix consisted of POCO, IG 430, C-2020, and SGL graphite grades. The Toyo-Tanso IG 430 is fine-grained isotropic graphite manufactured using the isostatic pressing method and made from coal tar pitch coke. It is considered an advanced grade because of the higher strength (37.2 MPa tensile and 90.2 compressive) as compared to IG 110 nuclear graphite grade. POCO graphite is a highly isotropic graphite (isotropy may vary from manufacturer to manufacturer) with typical hexagonal crystalline structure that exhibits higher strength than typical graphite. Variability between grades for as-received material was studied through offbeam tests for the thermal expansion coefficient in the range between room temperature and $300^{\circ} \mathrm{C}$, mechanical
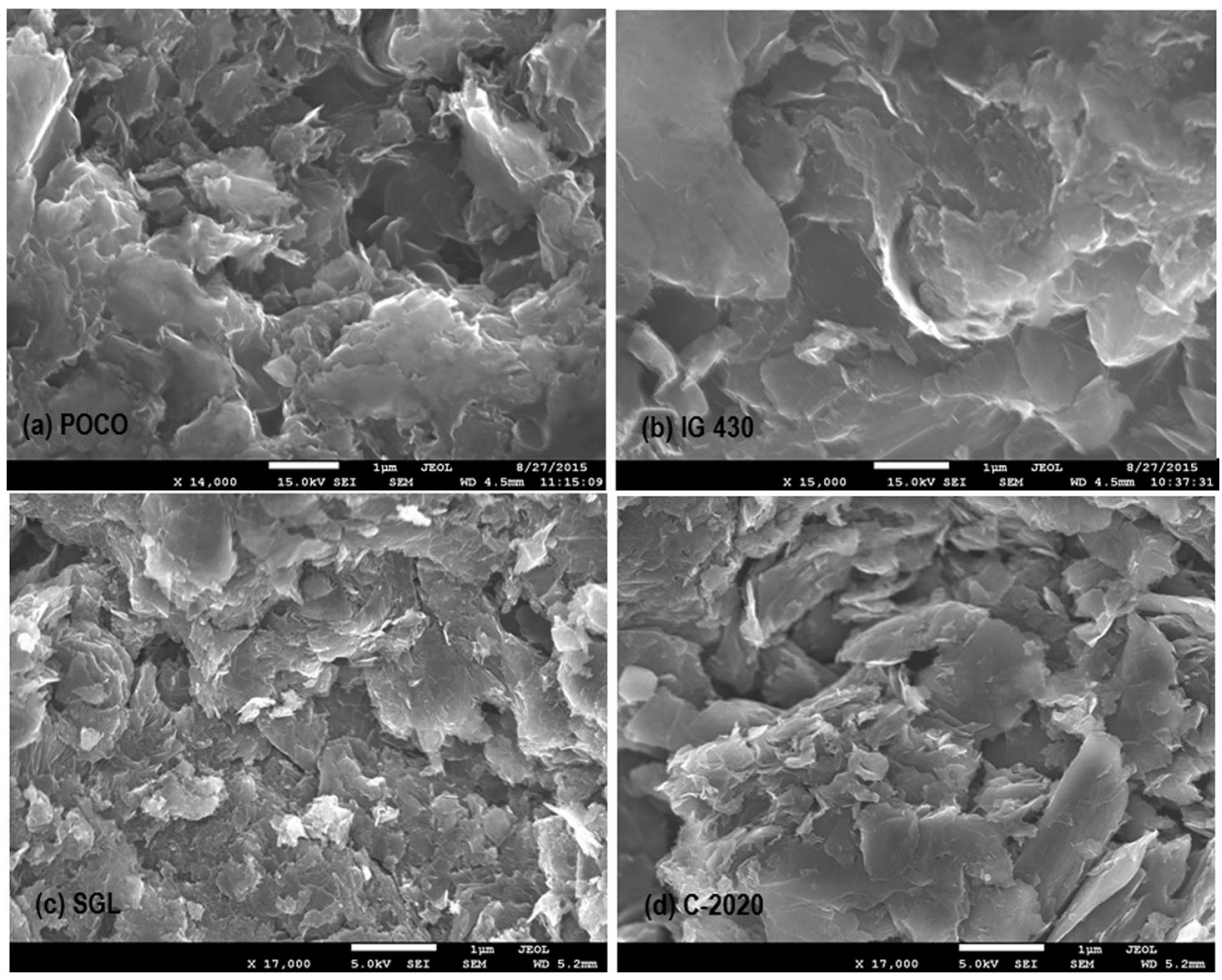

FIG. 1. Microstructural characterization and comparison of the four graphite grades using scanning electron microscopy. 

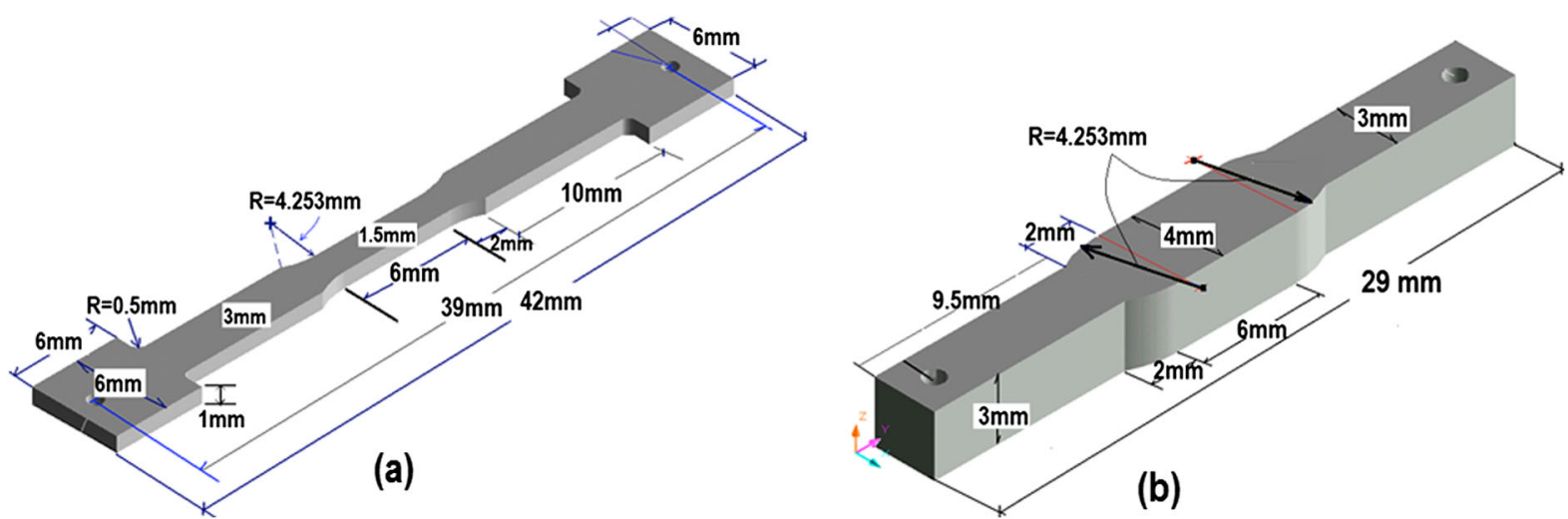

FIG. 2. Geometries of irradiated graphite specimens. Tensile sample (left) and CTE sample (right).
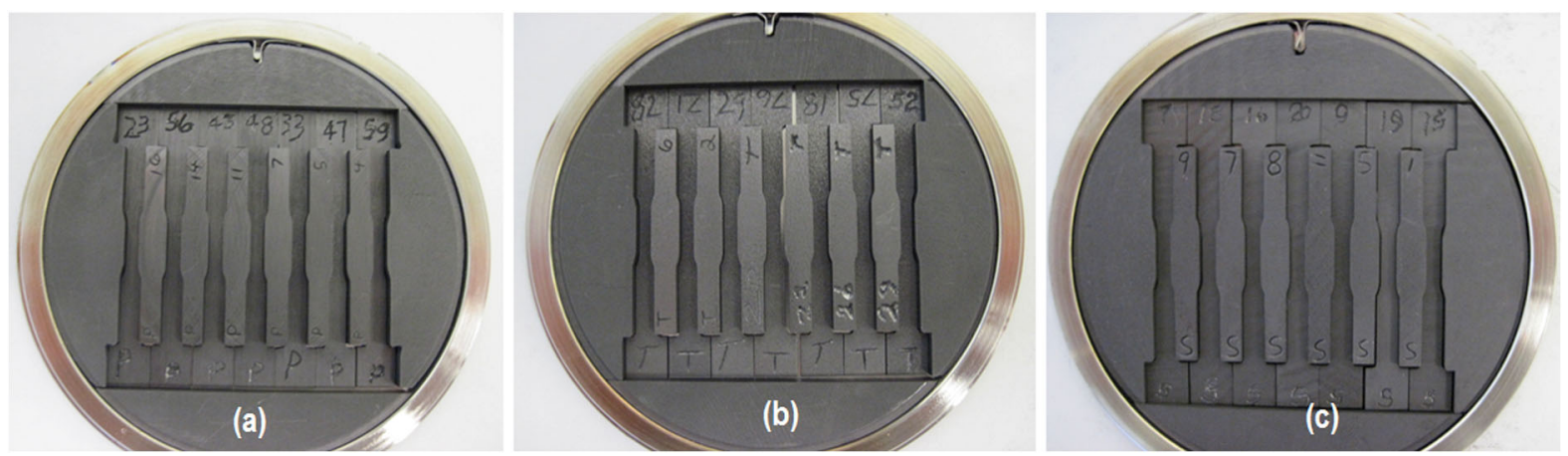

FIG. 3. Specimen layout within their respective argon-filled capsules of the LBNF graphite grades. (a) POCO, (b) IG 430, (c) SGL.

strength and microstructural characteristics using scanning electron microscopy. The microstructure of the unirradiated graphite was studied at the Center of Functional Nanomaterials (CFN) using scanning electron microscopy (SEM). Shown in Fig. 1 is microstructural comparison of the four graphite grades under study. C-2020 graphite [Fig. 1(d)] appears as the least orderly structure.

\section{A. Irradiation experiment sample preparation}

Two types of test specimens (shown in Fig. 2) were used for the irradiation and were arranged in a tight interweaved configuration (Fig. 3) in an effort to prevent gaps along the proton beam that will, if present, affect the quality of the beam profile downstream where isotope targets are irradiated simultaneously. Three (3) 1-mm thick tensile samples were stacked in each position for one 3-mm thick CTE sample. To enhance the heat removal from the contained samples to the cooling outside the capsule walls argon atmosphere was introduced in each capsule.

The overall irradiation configuration, including the isotope target array in the downstream irradiation position, is shown in Fig. 4. Also depicted in Fig. 4 is the proton beam profile through the capsules. A special Computational Fluid Dynamics (CFD) model simulating the geometry at the irradiation location where active water cooling was forced in the channels between the capsules has been formulated and run to establish convective heat transfer coefficients in the channels between the capsules, which in turn, was used along with energy deposition estimates by the proton beam on the graphite samples to estimate the irradiation temperature of each specimen in the array. Film coefficients of $\sim 7600 \mathrm{~W} / \mathrm{m}^{2}-\mathrm{K}$ were estimated

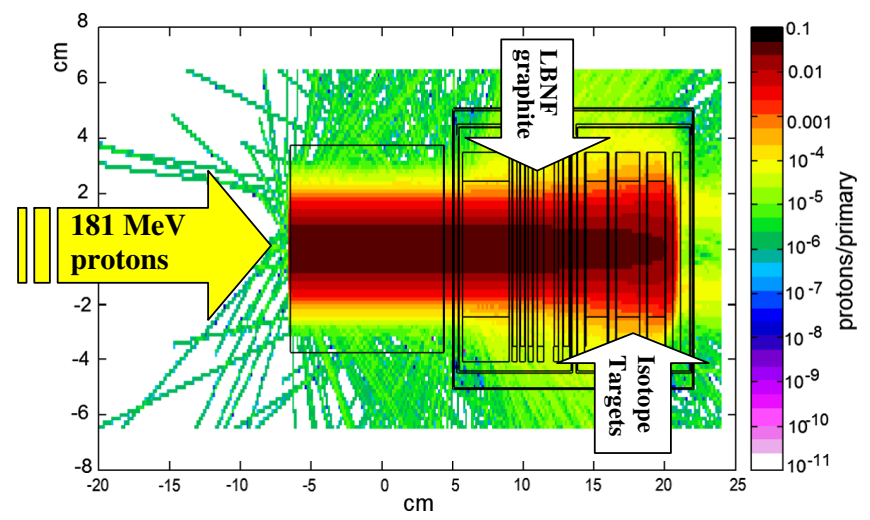

FIG. 4. Configuration of the $181 \mathrm{MeV}$ proton irradiation experiment of graphite grades. 

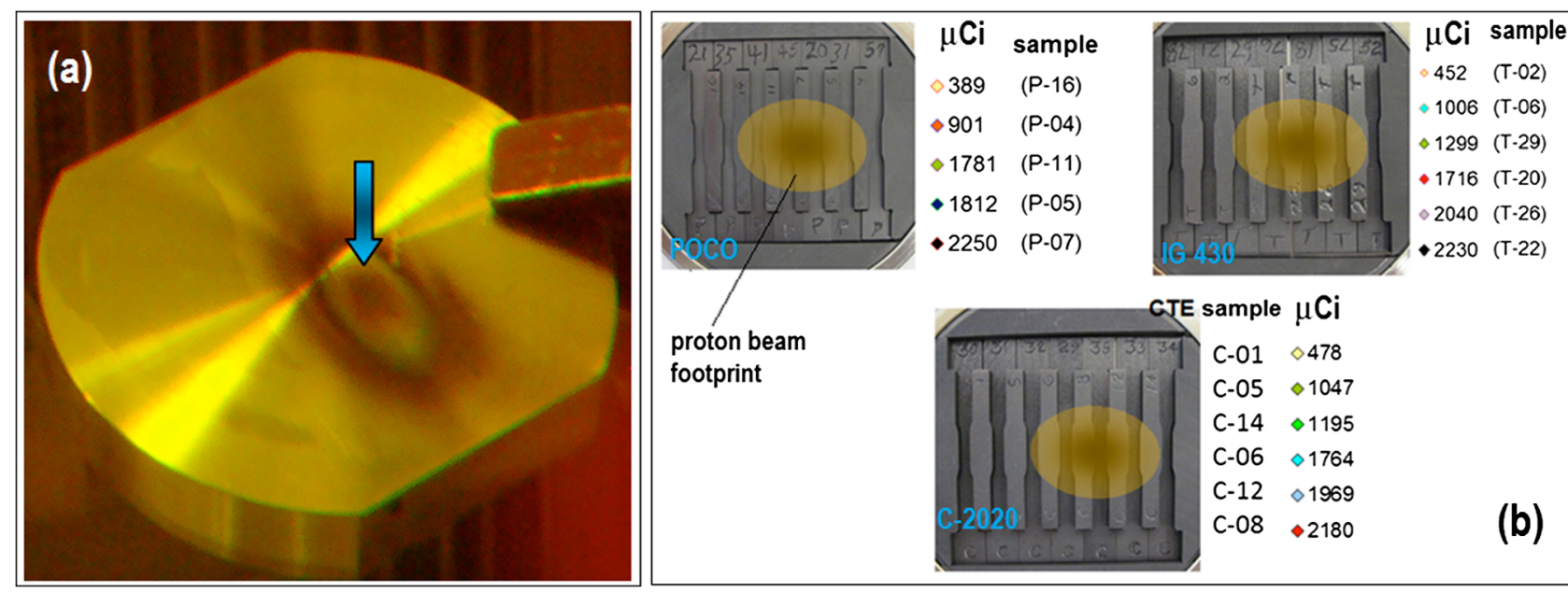

FIG. 5. (a) Imprint of proton beam on the stainless steel window of the vacuum capsule just upstream of the first graphite target capsule in the array, and (b) profile of activation measurements on the CTE-type samples contained in the target capsules conducted following irradiation.

based on coolant flow rate, temperature and channel geometry.

\section{B. Proton irradiation experiment}

The configuration shown in Fig. 4 was used for the irradiation. Due to the proton energy degradation through the different graphite capsules resulted in each grade of graphite being irradiated by slightly different proton energy. The proton energy ranged between $165-145 \mathrm{MeV}$, a variation not expected to have any significant impact on the irradiation damage. The total flux of protons incident on the LBNF target array was $3.247 \times 10^{21}$ (integrated beam current $\sim 144,310 \mu \mathrm{A}$-hours) and the beam profile was characterized by $\sigma_{x}=\sigma_{y} \sim 7.2 \mathrm{~mm}$. The 2D Gaussian profile of the proton beam was deduced from radiographic analysis of a Ni foil inserted in the array. The foil radiographic analysis revealed a beam offset quantified as $x_{o} \sim 4.0 \mathrm{~mm}$ (horizontal) and $y_{o} \sim 2.0 \mathrm{~mm}$ (vertical). The beam position was further confirmed by (a) the observation of the beam footprint on a vacuum capsule window just upstream of the graphite target array [Fig. 5(a)], and (b) actual activity measurements of the CTE-type samples of each of the graphite grades performed after irradiation [Fig. 5(b)]. Based on the integrated current of $3.247 \times 10^{21}$ protons, the Gaussian beam profile and the offset the fluence experienced by each of the samples (CTE or tensile) in the entire array were deduced. The estimated peak fluence for each of the four graphite grades was $\sim 6.1 \times 10^{20} \mathrm{p} / \mathrm{cm}^{2}$ experienced by the CTE sample located in the first right hand side position. The peak fluence over the 6-mm gauge (central, narrow section) of the tensile samples was estimated to be $\sim 5.95 \times 10^{20} \mathrm{p} / \mathrm{cm}^{2}$.

Because monitoring of the irradiation temperature during beam exposure was not feasible (a hermetically sealed irradiation configuration at the BLIP target station plus the added fact of graphite contained within capsules) irradiation temperatures were estimated based on an elaborate numerical model. Specifically, using the particle interaction and transport codes MARS [16] and FLUKA [17,18] the energy deposition throughout the array was estimated. Peak energy deposited by the proton beam on the graphite samples was $\sim 240 \mathrm{~W} / \mathrm{cm}^{3}$. Information on energy deposition was supplied to a finite element model which reproduced the enclosed graphite array of each capsule and, by considering the heat transfer coefficients estimated by the CFD model, the presence of argon gas and the heat transfer between specimens and capsule windows through contact, led to the temperature profile through the irradiated array. The thermal as well as the mechanical/deformation analyses were based on the capabilities of the LS-DYNA code [19]. The numerically estimated irradiation temperatures ranged between $120-200^{\circ} \mathrm{C}$. Therefore, between the estimated temperature and fluence profiles the irradiation temperature and corresponding proton fluence per sample was deduced throughout the array.

Following the estimation of the peak irradiation temperature of $\sim 200^{\circ} \mathrm{C}$, microcharacterization of the graphite grades was conducted by considering only the temperature parameter without the irradiation. Figure 6 depicts SEM images of SGL and C-2020 graphite grades after annealing in vacuum for 4 hours at $200^{\circ} \mathrm{C}$. Figure 6 (a) indicates the tendency of the SGL graphite towards a layered structure. In contrast, the microstructure of C-2020 remains less orderly. Therefore, any changes in the properties following bombardment are assessed to be due to the proton interaction with the polycrystalline graphite structures. A connection of the disorderly microstructure of C-2020 to the macroscopic stress-strain behavior under tension will be made in a later section. 

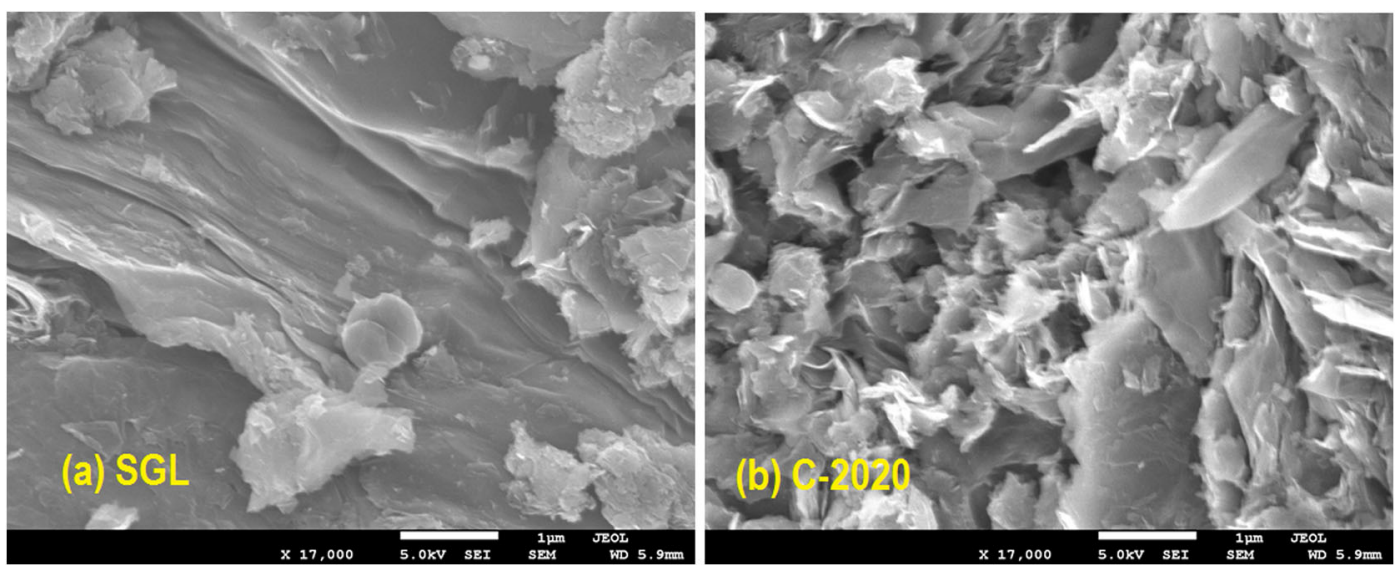

FIG. 6. SEM images of (a) SGL and (b) C-2020 graphite after annealing in vacuum at $200^{\circ} \mathrm{C}$.

\section{POSTIRRADIATION ANALYSES}

The postirradiation analysis of the four graphite grades consisted of (a) dimensional stability and thermal annealing as well as changes in thermal expansion coefficient (CTE), (b) mechanical testing to estimate radiationinduced changes in Young's modulus and strength and their recovery through postirradiation annealing and (c) x-ray diffraction using $200 \mathrm{KeV}$ "white beam" $x$ rays and EDXRD techniques at the BNL NSLS.

\section{A. Dimensional stability and thermal expansion coefficient}

Irradiation of graphite with energetic protons is expected to result in dimensional changes of considerable magnitude; an effect that must be accounted for in accelerator target design. Graphite can be viewed as an aggregate of crystallites of identical properties. The behavior of crystallite under irradiation is characterized by growth perpendicular to the basal planes and shrinkage parallel to the basal planes. For the aggregate, and in particular for isotropic graphite, the global effect is that the dimensional changes along the directions are not distinguishable. Porosity introduced during manufacturing of the graphite will experiences a reduction as graphite crystals experience irradiation-induced growth and grow into the preexisting pores. Therefore, deviations from the linear expansion of a graphite grade following irradiation will provide an indirect evidence of its porosity.

By utilizing a LINSEIS high resolution dilatometer the dimensional stability and thermal cycle annealing of the irradiated graphite grades were evaluated. Thermal cycling was conducted under one atmosphere pressure and in air. Figure 7 depicts the thermal expansion coefficient of the four, as-received grades and for the temperature range of $40-280^{\circ} \mathrm{C}$. As clearly shown, the CTE is stable up to $280^{\circ} \mathrm{C}$ for all four grades with the POCO graphite exhibiting a much higher expansion coefficient than the other three studied. CTE is an important target design parameter where lower values are desirable. More important, however, than the unirradiated CTE values is the quantification of the changes induced by proton irradiation to this important design parameter.

Shown in Fig. 8(a) are dimensional changes of the unirradiated graphite grades and in Fig. 8(b) dimensional changes after irradiation to the same fluence of $4.07 \times$ $10^{20} \mathrm{p} / \mathrm{cm}^{2}$ and $170{ }^{\circ} \mathrm{C}$ irradiation temperature. As seen in Fig. 8(b), the dimensional change of the irradiated graphite is linear up to the irradiation temperature of $170{ }^{\circ} \mathrm{C}$ stemming from the fact that annealing of radiation-induced defects is taking place simultaneously. During irradiation, interstitials accumulate between basal planes resulting in an overall volumetric growth of the polycrystalline structure. The contraction that is taking place is the result of annealing of interstitials that are further away from the lattice planes and require higher activation energy for annealing (return to unoccupied positions on the lattice plane). The higher isotropy graphite grades (IG 430 and POCO) exhibit the larger contraction due to thermal annealing.

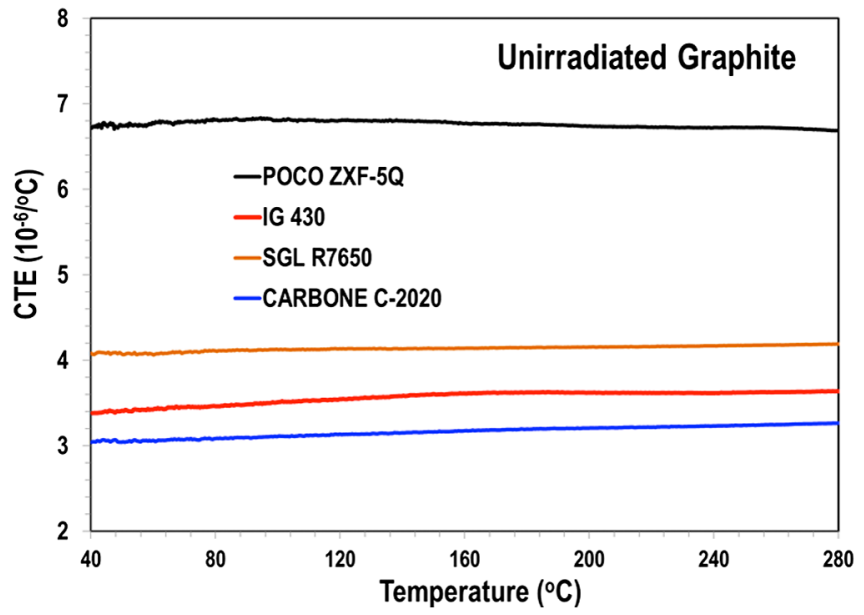

FIG. 7. Thermal expansion coefficient of as-received unirradiated graphite grades in the temperature range of $40-280^{\circ} \mathrm{C}$. 

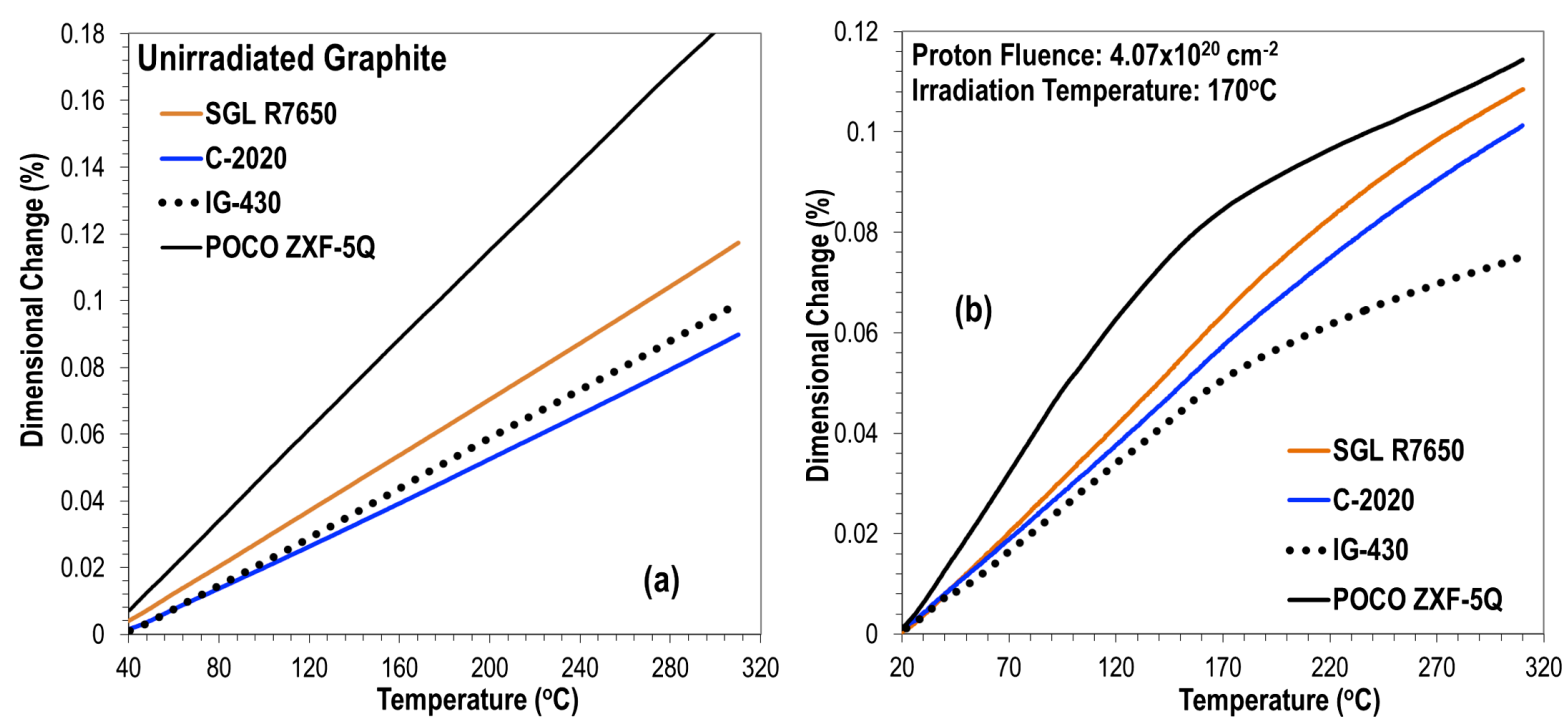

FIG. 8. Dimensional changes in as-received, unirradiated graphite grades (a) and following irradiation to the fluence of $4.07 \times 10^{20} \mathrm{p} / \mathrm{cm}^{2}$ at $170^{\circ} \mathrm{C}$. Heating rate of $2^{\circ} \mathrm{C} /$ minute was used.

Thermal cycle annealing of all four irradiated grades of graphite indicated that annealing of interstitial defects produced during irradiation occurs primarily within the first thermal cycle. Shown in Fig. 9 (left) is postirradiation thermal annealing of irradiated POCO graphite to the peak fluence $\left(6.1 \times 10^{20} \mathrm{p} / \mathrm{cm}^{2}\right)$ at $200^{\circ} \mathrm{C}$. During the first thermal cycle irradiation damage is annealed as indicated by the contraction behavior. Subsequent cycles reveal that the slope of the dimensional change increased (change of thermal expansion coefficient) but with no changes occurring after the first cycle.

Figure 9 (right) depicts postirradiation annealing of IG 430 graphite for two fluence levels and corresponding

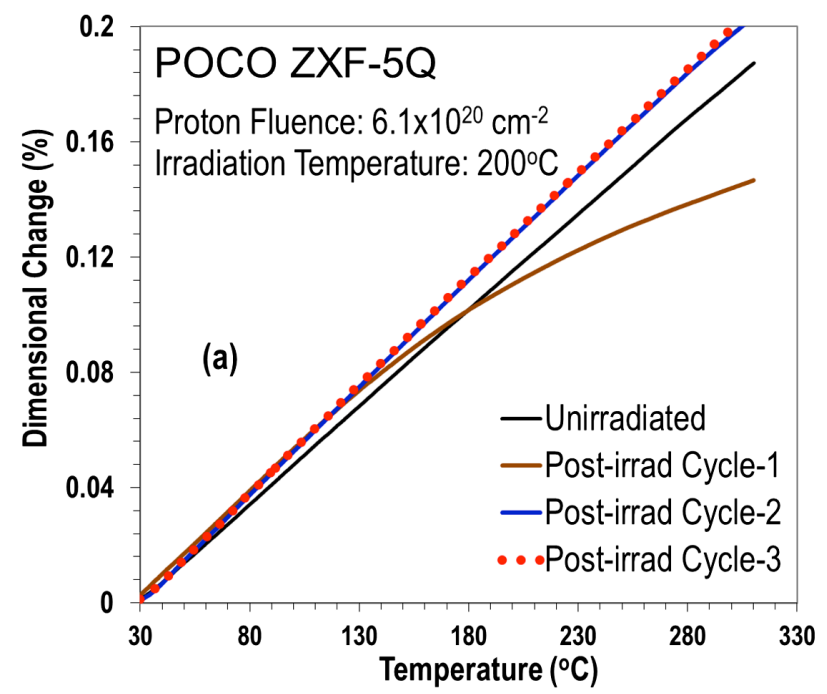

irradiation temperatures. It is apparent that annealing of the two different irradiated conditions leads to similar slope changes that are indistinguishable. It is important to note, however, that the volumetric change (growth) induced during irradiation is higher for the lower fluence-lower temperature. This confirms reported results on graphite growth rates [4] for fast neutron irradiation where growth rates change by a factor of 8 between $80^{\circ} \mathrm{C}$ and $220^{\circ} \mathrm{C}$ (higher at $80^{\circ}$ ). It is therefore assessed that it is the irradiation temperature that is more responsible for the higher growth observed than the proton fluence received. All four grades exhibited similar behavior regarding irradiation growth due to temperature and fluence.

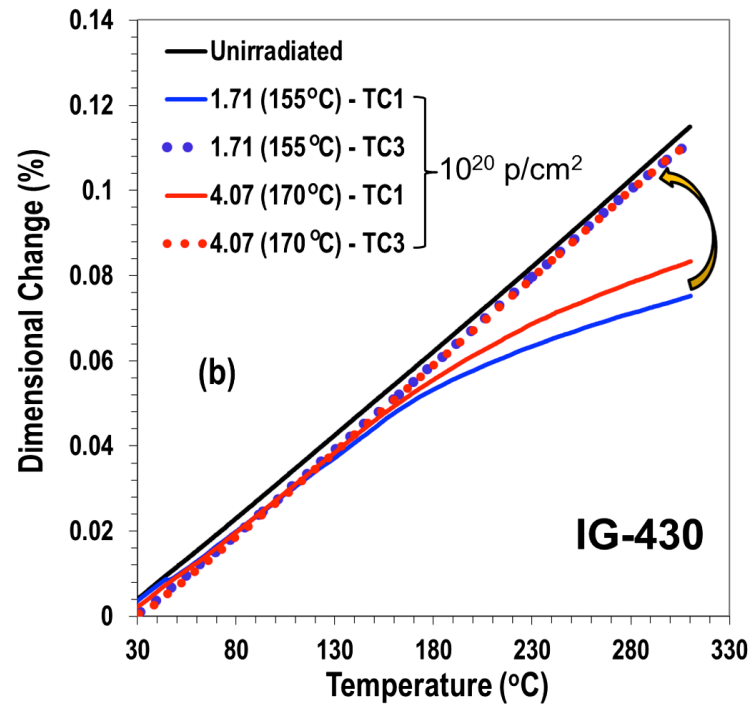

FIG. 9. (a) Thermal cycle annealing of irradiated POCO graphite over three postirradiation cycles to $310^{\circ} \mathrm{C}$ to fluence of $6.1 \times 10^{20} \mathrm{p} / \mathrm{cm}^{2}$ and irradiation temperature of $200^{\circ} \mathrm{C}$ and (b) postirradiation annealing of IG 430 irradiated to two fluence levels and corresponding irradiation temperatures. 

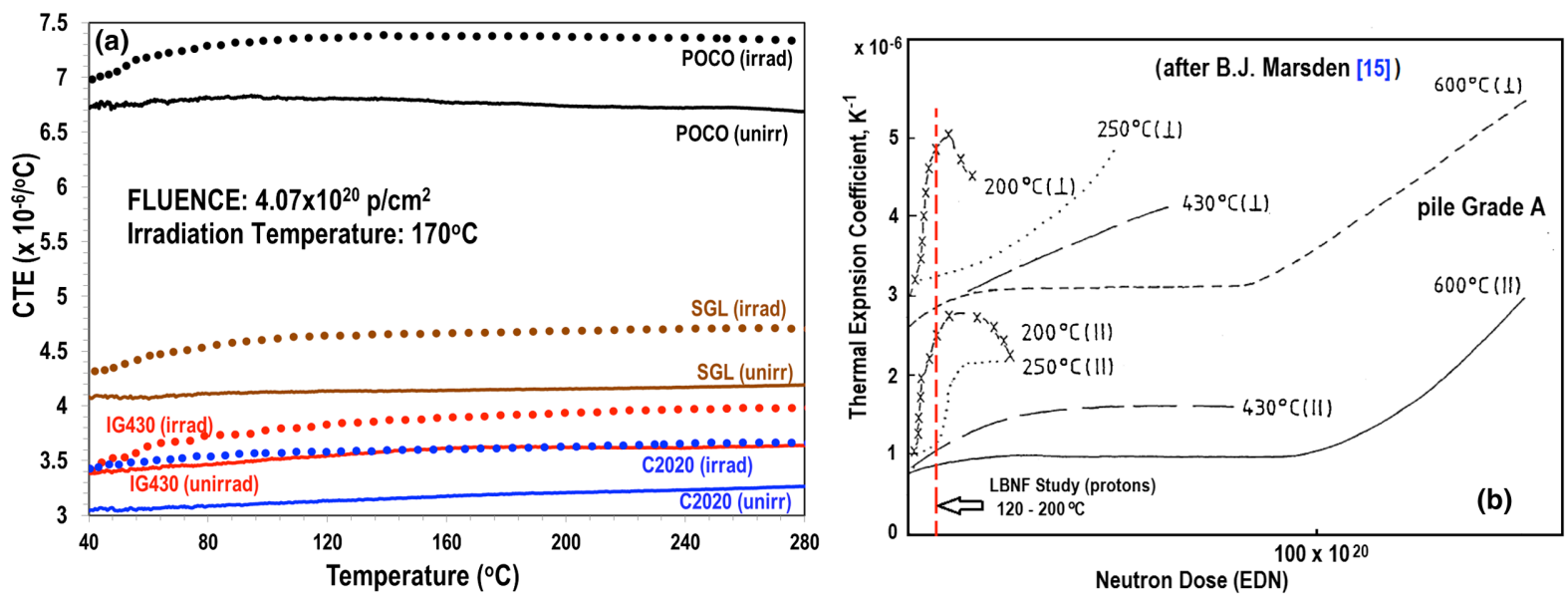

FIG. 10. Irradiation-induced changes in the CTE. (a) LBNF graphite grades irradiated at $170{ }^{\circ} \mathrm{C}$ and (b) pile grade A graphite (after Marsden [11]).

Depicted in Fig. 10(a) are CTEs for all four graphite grades of graphite studied and irradiated to the same fluence and at same irradiation temperature $\left(4.07 \times 10^{20} \mathrm{p} / \mathrm{cm}^{2}\right.$ and $170{ }^{\circ} \mathrm{C}$ respectively). All four grades exhibit an increase of CTE due to proton irradiation. Figure 10(b) (after
Marsden [11]) shows change of CTE induced by neutron irradiation at different temperatures, including temperatures as low as $200^{\circ} \mathrm{C}$. Shown in Fig. 10 (b) is the increase of CTE occurring in irradiated pile graphite for the lower temperatures exposed to neutron fluences
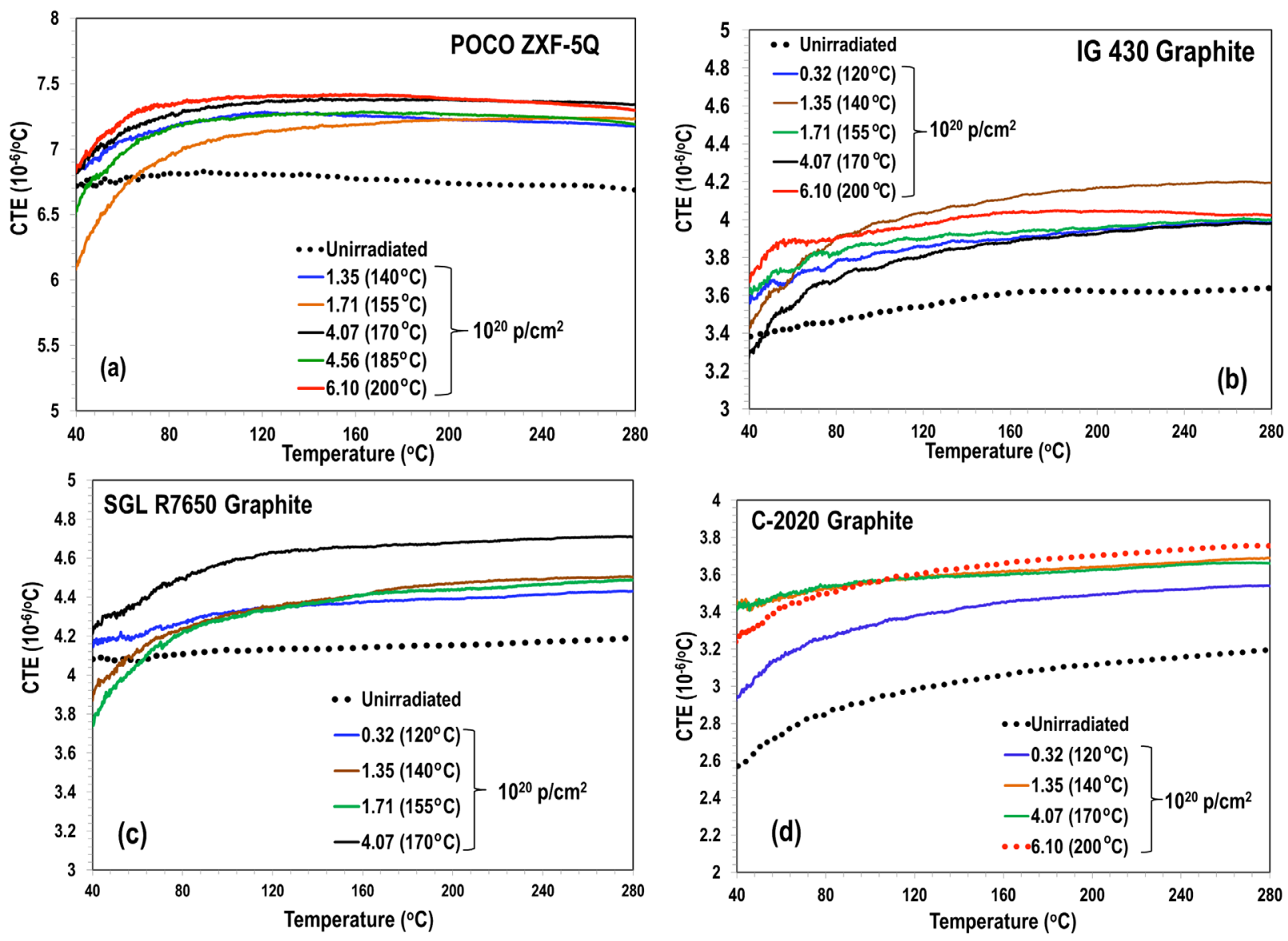

FIG. 11. Proton fluence effects on thermal expansion coefficient for (a) POCO, (b) IG 430, (c) SGL and (d) C-2020 graphite grades. Indicated are fluence followed by corresponding irradiation temperature. 
similar to the proton fluence and temperatures of this study.

Figure 11 shows the influence of proton fluence on the CTE of three of the four graphite grades studied (POCO, IG 430 and SGL). As noted in Fig. 11, while the CTE is shown to increase for all fluence levels depicted, there exists variation between the grades regarding the fluencetemperature combination effect on CTE. Looking closer at POCO graphite [Fig. 11(a)], it is evident that the CTE has reached a maximum in the fluence range of 4.07-6.01 $\times$ $10^{20} \mathrm{p} / \mathrm{cm}^{2}$. As reported in [3], for works of Simons, Kelly, etc., for neutron irradiation at room temperature the CTE reaches a maximum at $\sim 5 \times 10^{20} \mathrm{n} / \mathrm{cm}^{20}$ while at $225^{\circ} \mathrm{C}$ the dose threshold where CTE reaches a maximum and decrease starts is lower at $\sim 4.0 \times 10^{20} \mathrm{n} / \mathrm{cm}^{20}$. A similar trend has been observed here for graphite under proton irradiation.

This experimentally verified similarity of irradiationinduced changes between protons and fast neutrons is of paramount importance towards the estimation of the behavior of the explored four graphite grades at higher than achieved fluence levels. Neutron-based irradiation effects on graphite CTE available at higher fluences and over different irradiation temperatures stemming from several decades of research and reactor operation experience can be utilized as a guide in selecting operating temperatures and extrapolating to higher fluences than those achieved during proton irradiation experiments.

\section{B. Irradiation effects on the mechanical properties and strength}

The effect of proton irradiation on the strength and Young's modulus of the four graphite grades was studied using a $5 \mathrm{kN}$ tensile test apparatus (Tenius-Olsen). The tensile tests were conducted in room temperature. As noted previously, during irradiation individual graphite crystals undergoing irradiation growth grow into and fill the pores introduced during manufacturing leading to a "tightening up" of the aggregate. This is expected to result in an increase of the elastic modulus and in conjunction with dislocation pinning into an increase of the strength. Studies of neutron-irradiated graphite [3] indicated that Young's
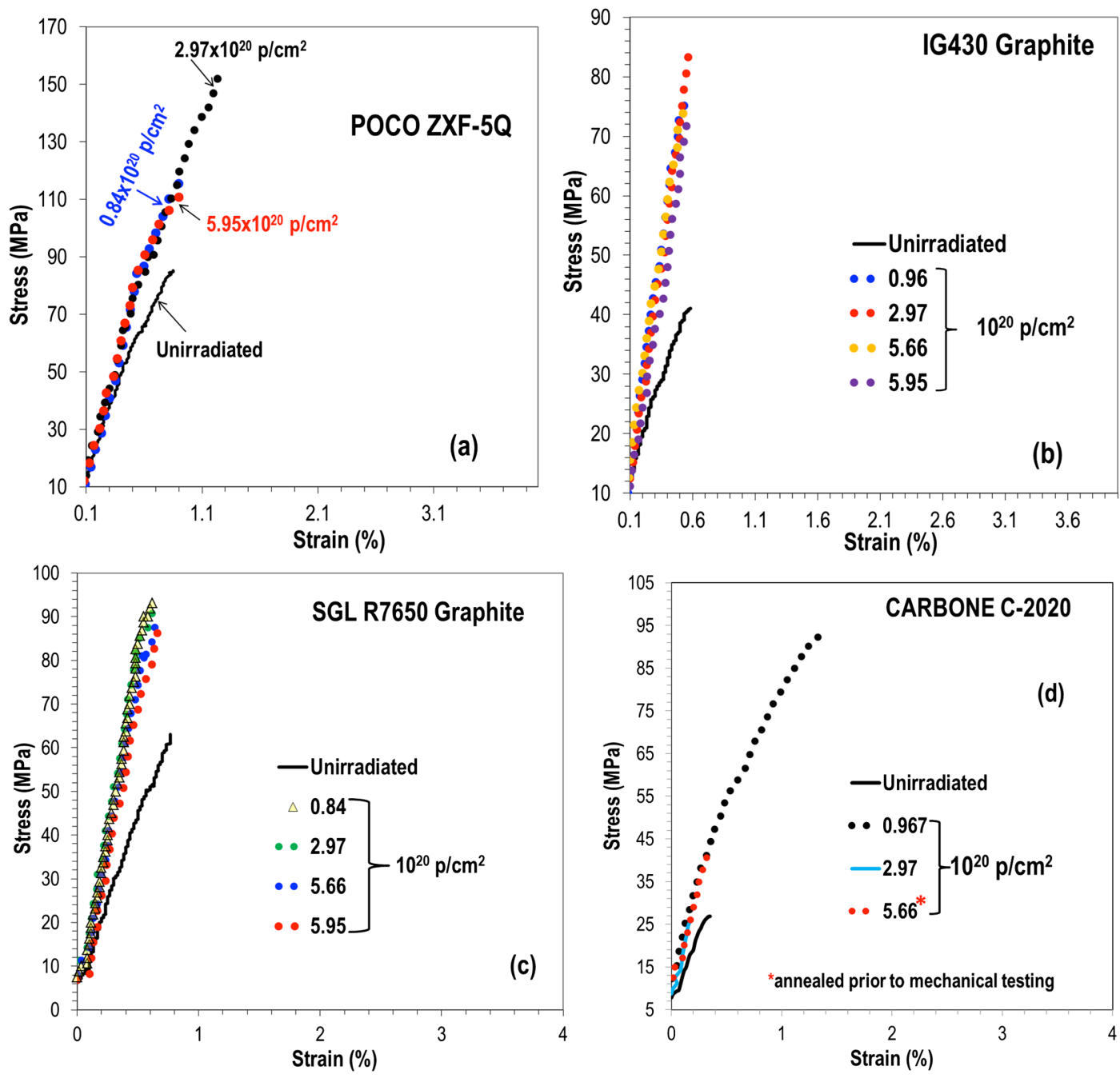

FIG. 12. Stress-strain behavior of proton irradiated (a) POCO, (b) IG 430, (c) SGL and (d) C-2020 graphite grades. 
modulus and strength are affected by irradiation and experience increase. As reported in [3] irradiation at room temperature to fluence of $10^{20} \mathrm{n} / \mathrm{cm}^{2}$ leads to a maximum in the values of modulus and strength. At $10^{21} \mathrm{n} / \mathrm{cm}^{2}$ Young's modulus has been shown to increase by a factor of 3 above the unirradiated value while at higher irradiation temperatures the changes observed are smaller.

In the experiments conducted and reported here the irradiating species were energetic protons instead of neutrons; the operating temperature was in the range of $120-200^{\circ} \mathrm{C}$ and the peak fluence $\sim 6.0 \times 10^{20} \mathrm{p} / \mathrm{cm}^{2}$. The objective of the postirradiation mechanical testing of the four grades was not only to compare the mechanical behavior and strength evolution of the four grades with irradiation but to also compare the effects with those observed under neutron irradiation. Qualitative agreement in the behavior of graphite between neutron and proton irradiation will permit the use of neutron data as a guide to higher proton fluences in combination with other regimes of irradiation temperature.

Shown in Fig. 12 are tensile test results conducted on the tensile specimens shown in Fig. 2 for the four irradiated grades. The effect of the received fluence on the strength and the elastic modulus (slope of the stress-strain curve) is shown by comparing results with the unirradiated graphite. The following observations can be made regarding the results of Fig. 12.

POCO exhibited the highest strength in the unirradiated state ( $90 \mathrm{MPa})$ followed by the SGL, IG 430 and finally C-2020. The higher strength of POCO graphite is attributed to the low porosity achieved during its fabrication and its high isotropy. SGL graphite showed the least scattering in unirradiated strength and Young's modulus while C-2020 the highest.

Irradiation results in significant increases of both strength and Young's modulus for all four grades. For the highly isotropic graphite grades (POCO and IG 430) the strength increases by $\sim 80 \%$ and $\sim 100 \%$ respectively. The Young's modulus on the other hand increases by $\sim 60 \%$ for POCO and $140 \%$ for IG 430. For SGL graphite the increase in strength is $\sim 50 \%$ and the Young's modulus increased by almost a factor $2(\sim 92 \%)$. C-2020 graphite experiences the highest increase in strength (factor of $\sim 3$ ) and significant variation between fluence levels [partially influenced by the scattering of the data observed during tensile tests of the unirradiated samples as shown in Fig. 13(a)].

Important to note from qualitatively assessing these mechanical tests is that the strength reaches a maximum at a similar fluence $\left(2.97 \times 10^{20} \mathrm{p} / \mathrm{cm}^{2}\right)$ for two of the grades (POCO, IG 430) followed by a decrease at higher fluence. The SGL graphite strength appears to reach the maximum strength at a lower fluence $\left(0.84 \times 10^{20} \mathrm{p} / \mathrm{cm}^{2}\right)$ but with less drastic reduction of strength at higher fluences, as if it is reaching a plateau. For C-2020 this trend is erratic with maximum strength experienced at $\sim 1.0 \times 10^{20} \mathrm{p} / \mathrm{cm}^{2}$. The erratic nature of C-2020 mechanical behavior is attributed to its microstructure and is further supported by the wide scattering of the mechanical data of the unirradiated material depicted in Figure 13(a). These observations on the irradiation-induced changes in graphite strength, being a function of both the graphite grade and fluence, are of paramount importance in both the selection of a graphite grade for the future LBNF target and for the actual design in that fluence thresholds for maximum strength and Young's modulus increase have been identified. This significant finding is in full agreement with the observation made on the mechanical behavior of graphite under neutron irradiation. Specifically, based on results presented in [3] the strength and Young's modulus of graphite irradiated with neutrons increase up until $\sim 10^{20} \mathrm{n} / \mathrm{cm}^{2}$ for irradiation performed at room temperature and they start decreasing at higher fluences with changes being smaller at higher temperatures. Also reported in [11] for Pile Grade A and Gilsocarbon graphite grades under neutron irradiation is the presence of two distinct regions about the $300^{\circ} \mathrm{C}$ irradiation temperature. Below $300^{\circ} \mathrm{C}$ the Young's modulus increases by a factor of
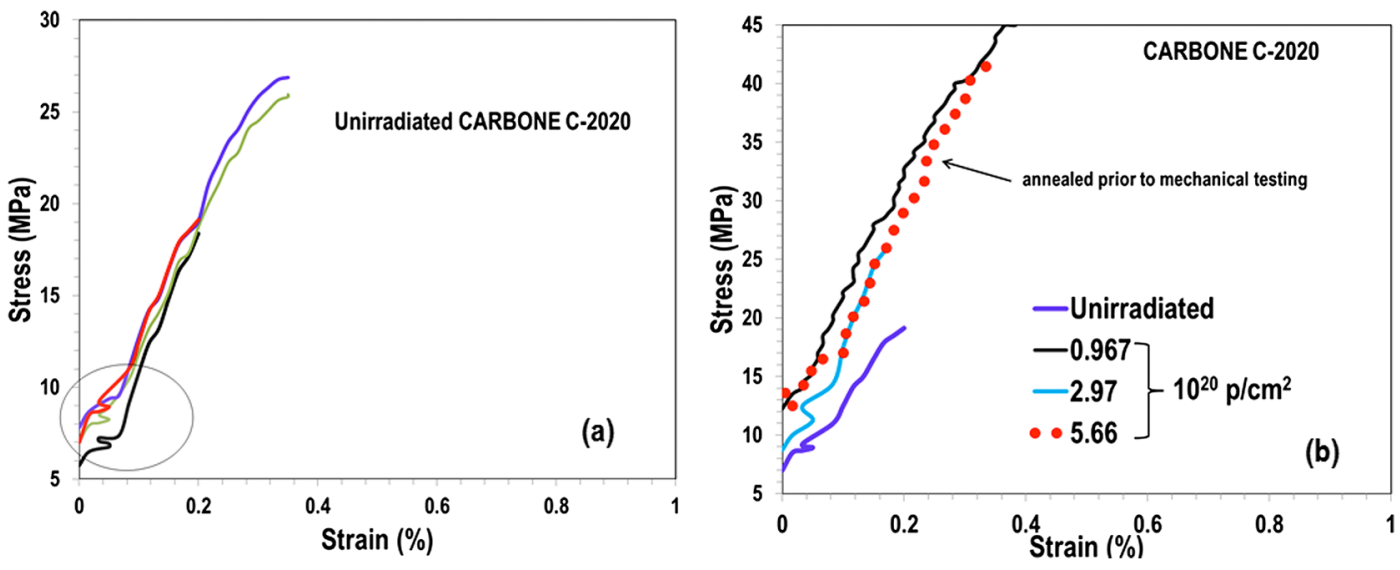

FIG. 13. Mechanical behavior of (a) unirradiated and (b) irradiated CARBONE C-2020 graphite. 


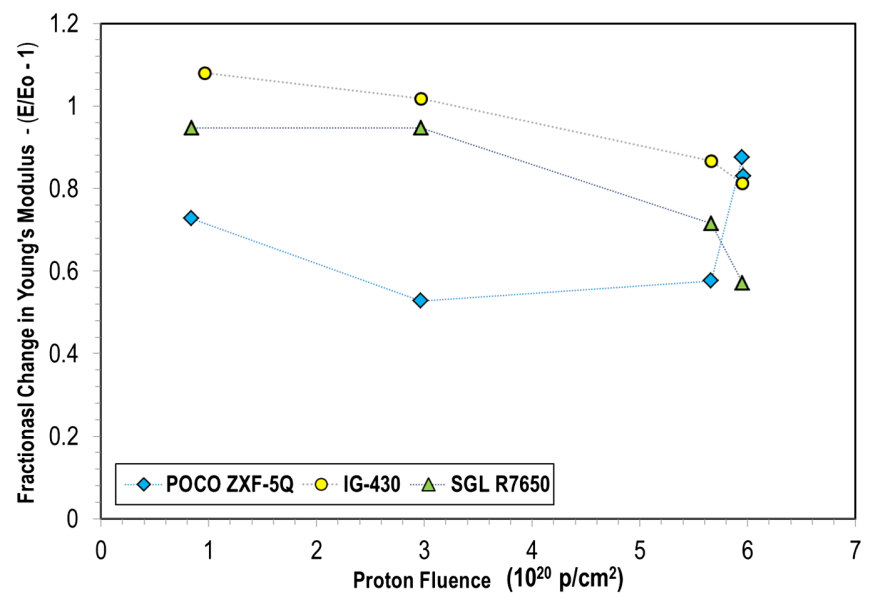

FIG. 14. Measured fractional change in the Young's modulus of three graphite grades, POCO, IG 430 and SGL, as a function of proton fluence.

$\sim 3$, peaks and then decreases, followed by an increase and later a catastrophic decrease and disintegration. The explanation for this behavior is that, for low doses at temperatures below $300{ }^{\circ} \mathrm{C}$, pinning of the dislocations leads to an increase of modulus. The decrease in modulus that follows is due to large separation in the basal plane induced by higher neutron irradiation. Peak values of Young's modulus at irradiation temperatures $<300^{\circ} \mathrm{C}$ occur at fluence in the range of $1-2 \times 10^{20} \mathrm{n} / \mathrm{cm}^{2}$ depending on the temperature. The reported behavior of graphite in [11] under neutron irradiation agrees with the findings of the present study under proton irradiation.

Shown in Fig. 13 is the mechanical behavior of C-2020 during tensile tests. Figure 13(a) depicts the stress-strain response of samples of unirradiated C-2020 graphite. As noted previously, the grade exhibits wide scattering of the data both in terms of strength and Young's modulus, the latter based on the slope of the stress-strain curve. The strength of C-2020 is the lowest amongst the four grades studied. Interesting to note is the presence of a "kink" at low tensile stress, an indication that the structure is "adjusting" and eventually locking up. This behavior under tension at the early stages of the stress-strain curve may be explained by the micrographs generated for this graphite grade at room temperature and $200{ }^{\circ} \mathrm{C}$ [Figs. 1(d) and 6(b) respectively] which show a greater degree of disorder as compared to the other three grades.

Based on Fig. 12(d) the strength increases by more than a factor greater than 3 but at lower fluence as compared to the other three grades. At higher fluences there appears to be significant reduction in strength [Figs. 12(d) and 13(b)]. The "kink" exhibited for the unirradiated C-2020, as shown in Fig. 13(b), is still observed following irradiation as well as postirradiation annealing to $350{ }^{\circ} \mathrm{C}$. This characteristic behavior may be attributed to the "disordered" microstructure observed under the microscope [Figs. 1(d) and 6(b)] which "locks up" upon tensile load application. Due to the wide scattering of the data that apparently stem from the "unsettling" of the microstructure during manufacturing, the low strength and the different behavior under irradiation, as compared to the three other grades, the C-2020 was removed from further consideration and in particular microstructural studies (x-ray diffraction) reported in the next section. Figure 14 depicts the fractional change of Young's modulus for the three remaining graphite grades (POCO, IG 430 and SGL) as a function of proton fluence.
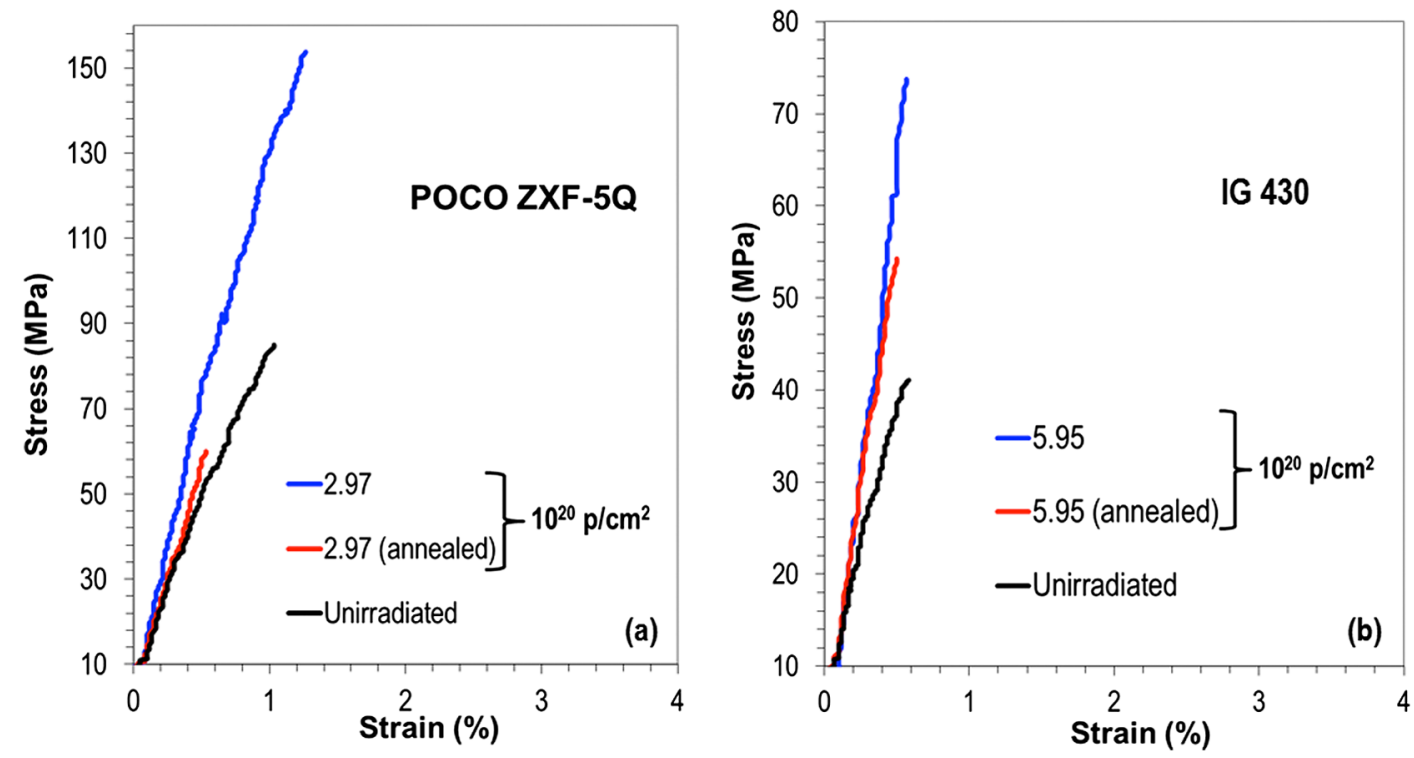

FIG. 15. Postirradiation annealing effects on strength and Young's modulus of (a) POCO and (b) IG 430 graphite grades. 

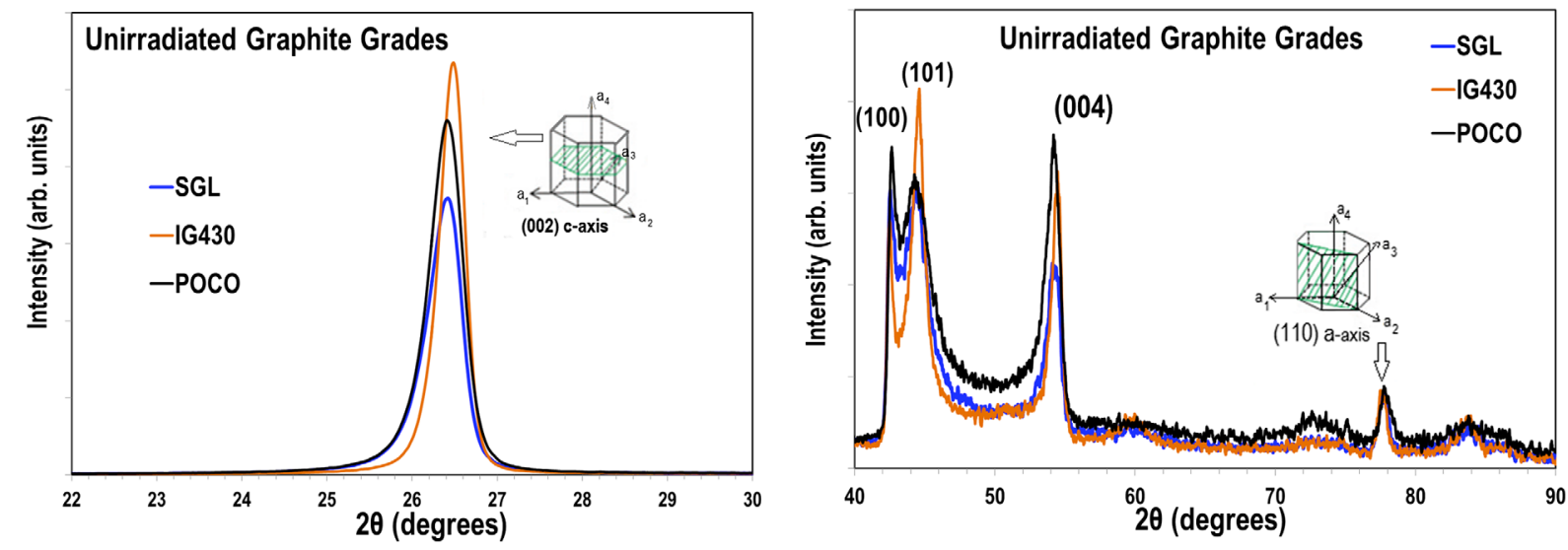

FIG. 16. X-ray diffraction of as-received, unirradiated graphite grades POCO, IG 430 and SGL.
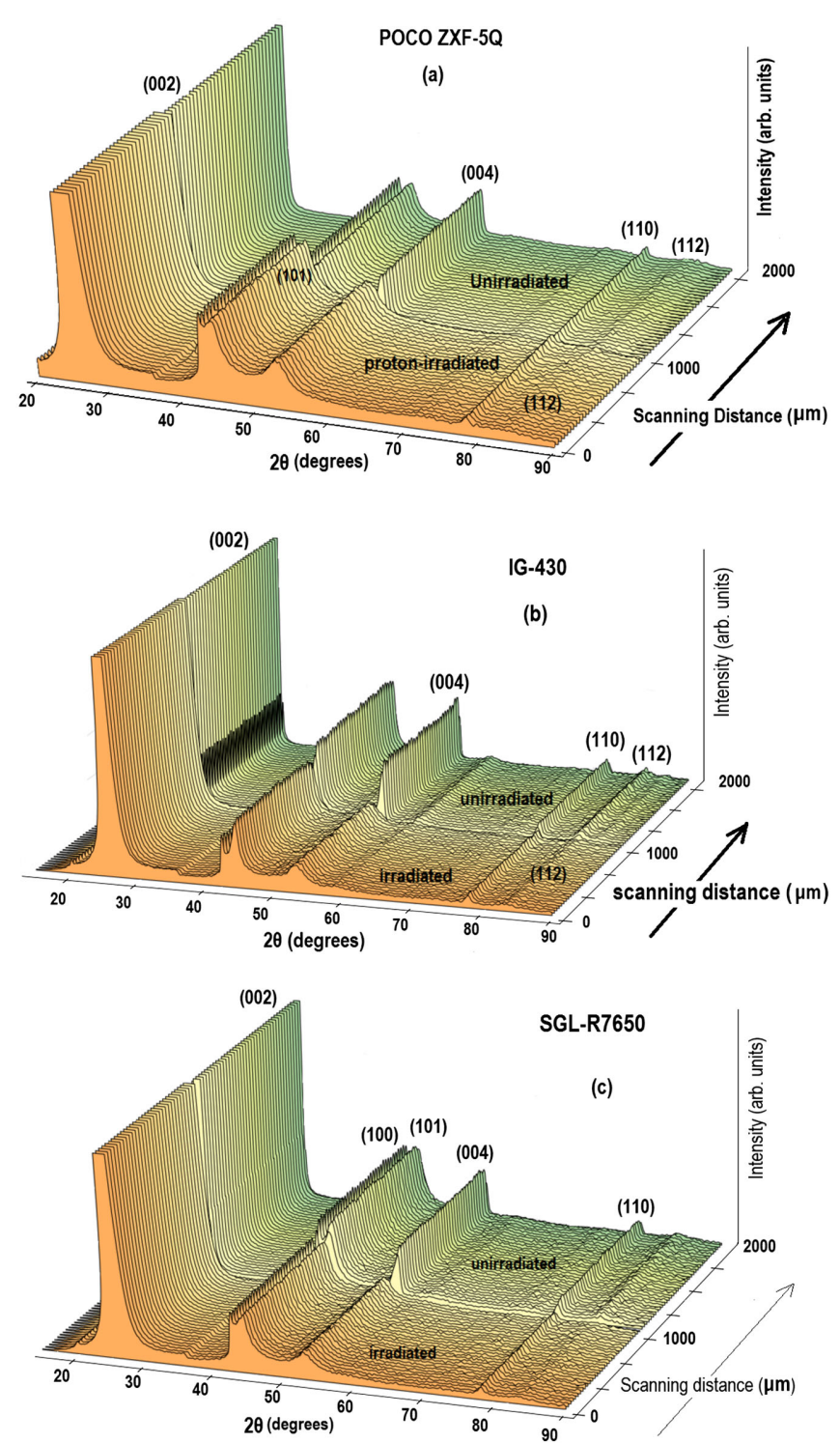

FIG. 17. 3D X-ray phase map of proton-irradiated (a) POCO, (b) IG 430 and (c) SGL graphite grades for proton fluence of $5.66 \times 10^{20} \mathrm{p} / \mathrm{cm}^{2}$. (002) diffraction peak is truncated for clarity.

\section{Annealing effects on irradiated Graphite Young' modulus and strength}

According to data reported in [5] for neutron irradiated graphite, the "tightening up" of the aggregate structure of graphite crystals due to irradiation-induced growth into preexisting pores results in the increase of Young's modulus. This increase can only partially be annealed out. It is pointed out [5] that while the postirradiation thermal anneals and the resulting recovery has no satisfactory explanation, the data nevertheless are of direct utility to designers. Postirradiation annealing to $350^{\circ} \mathrm{C}$ of tensile samples irradiated with protons confirmed the findings in [3] of partial restoration of Young's modulus as seen in Fig. 15 for POCO and IG 430. The partial restoration is accompanied by a significant reduction in the acquired strength during irradiation.

\section{Microstructural evaluation based on $x$-ray diffraction of irradiated LBNF graphite grades}

In an effort to further explore differences and similarities between graphite grades and the influence of proton irradiation on their microstructure $\mathrm{x}$-ray diffraction was conducted using $200 \mathrm{keV}$ white $\mathrm{x}$ rays and EDXRD techniques at the X17B1 beam line of the NSLS synchrotron. The damage induced by proton irradiation originates at the microstructure or lattice of graphite and manifests itself into macroscopic property changes that were observed and presented in the previous sections.

Figure 16 compares $\mathrm{x}$-ray diffraction results obtained for the as-received graphite for POCO, IG 430 and SGL grades. Of particular interest are the diffraction peaks (002) and (110) that define the two principal axes in the graphite lattice, $c$-axis normal to the basal planes and $a$-axis parallel to the basal planes. In addition, the shape of the (002) diffraction peak and in particular the spacing between the basal planes $\left(\mathrm{d}_{002}\right)$ also provides an indication of the degree of graphitization achieved during manufacturing. The study on unirradiated graphite revealed that the d-spacing $\mathrm{d}_{002}$ 


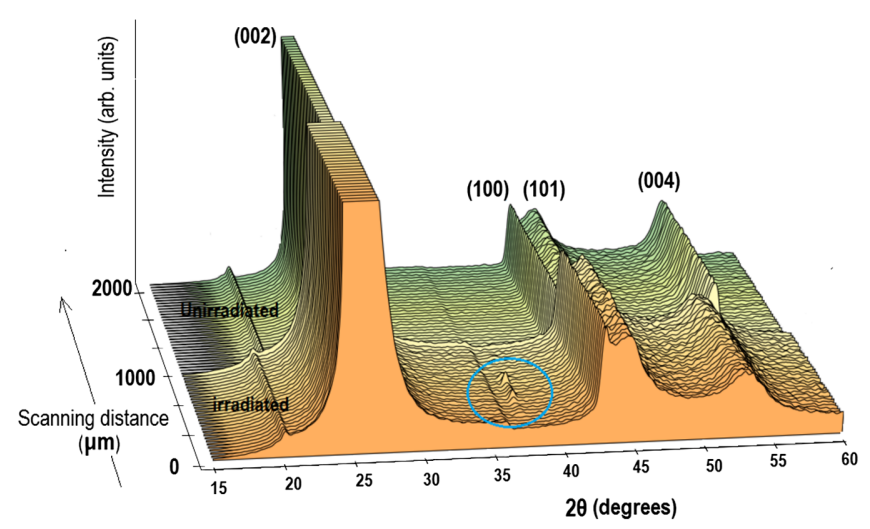

FIG. 18. XRD map of irradiated POCO graphite around the (002) diffraction peak and the effect of $5.66 \times 10^{20} \mathrm{p} / \mathrm{cm}^{2}$ fluence. (002) diffraction peak is truncated for clarity.

is $3.3833 \AA$ for POCO, $\mathrm{d}_{002}=3.37944 \AA$ for SGL R7650 and $\mathrm{d}_{002}=3.36814 \AA$ for Toyo-Tanso IG 430. The ideal graphite crystallite is characterized by $\mathrm{d}_{002}=3.3354 \AA$. The narrower (002) diffraction peak shape for IG 430 is a further indication of the higher graphitization achieved during its manufacturing. Less variability between the grades is observed in the $a$ lattice parameter where a equals $1.227 \AA, 1.230 \AA$ and $1.229 \AA$ for POCO, IG 430 and SGL respectively.

Figure 17 shows 3D phase maps of irradiated graphite compared to the unirradiated state. X-ray scanning was performed across the $1 \mathrm{~mm}$ thickness of the irradiated tensile samples shown in Fig. 2. The effects of proton irradiation of $5.66 \times 10^{20} \mathrm{p} / \mathrm{cm}^{2}$ are clearly shown with shifts, accompanied by widening, of the (002) and (004) diffraction peaks towards smaller diffraction angles and of the (110) peak associated with the $a$-axis parallel to the basal plane shifting towards higher diffraction angles. What is important to note is the almost absent diffraction of the twinning graphite crystal plane (112) in POCO graphite. The (112) crystallographic plane is clearly delineated in IG 430 but less so in RGL R7650. For all three grades, however, and with the onset of irradiation, the (112) diffraction peak begins to disappear. This is attributed to the formation of interstitial clusters between the basal planes. Figure 18 explores the diffraction map near the (002) peak for POCO graphite which appears to be the only one of the three grades where new crystalline planes are formed and the graphite becomes turbostratic.

The $c$-axis growth for the three grades following irradiation to $5.6 \times 10^{20} \mathrm{p} / \mathrm{cm}^{2}$ fluence is deduced from the EDXRD data and are $\sim 3.154 \%$ for POCO, $\sim 2.9 \%$ for R7650 and $\sim 2.83 \%$ for IG 430 . At fluence $\sim 6 \times 10^{20} \mathrm{p} / \mathrm{cm}^{2}$ the $c$-axis growth in IG 430 graphite increased to $\sim 4.15 \%$ while the $a$-axis shrank by $\sim 0.51 \%$, the latter being the result of vacancies forming on the basal planes of graphite.

Figure 19 depicts dimensional changes along the $c$-axis and $a$-axis of proton irradiated graphite grades deduced from this study. Shown in Fig. 20 are dimensional changes along the $c$ and $a$ axes for neutron irradiated pyrolytic graphite reported by Marsden [11] as a function of irradiation temperature and neutron fluence. As seen in Fig. 20 two irradiation temperature regimes are clearly delineated, one below $200^{\circ} \mathrm{C}$ and one above. The $c$ and $a$ axes changes triggered by proton irradiation and deduced from the energy dispersive x-ray diffraction (EDXRD) study agree generally well with the corresponding neutron irradiation data for the temperature range of $120-200{ }^{\circ} \mathrm{C}$.

It has been assessed through macroscopic studies that the fluence of $5.0 \times 10^{20} \mathrm{p} / \mathrm{cm}^{2}$ represents an irradiation damage threshold for graphite. This, in addition to its demonstration in the current study, has also been shown to occur under neutrons. The similar behavior in CTE, strength and Young's modulus evolution with fluence,
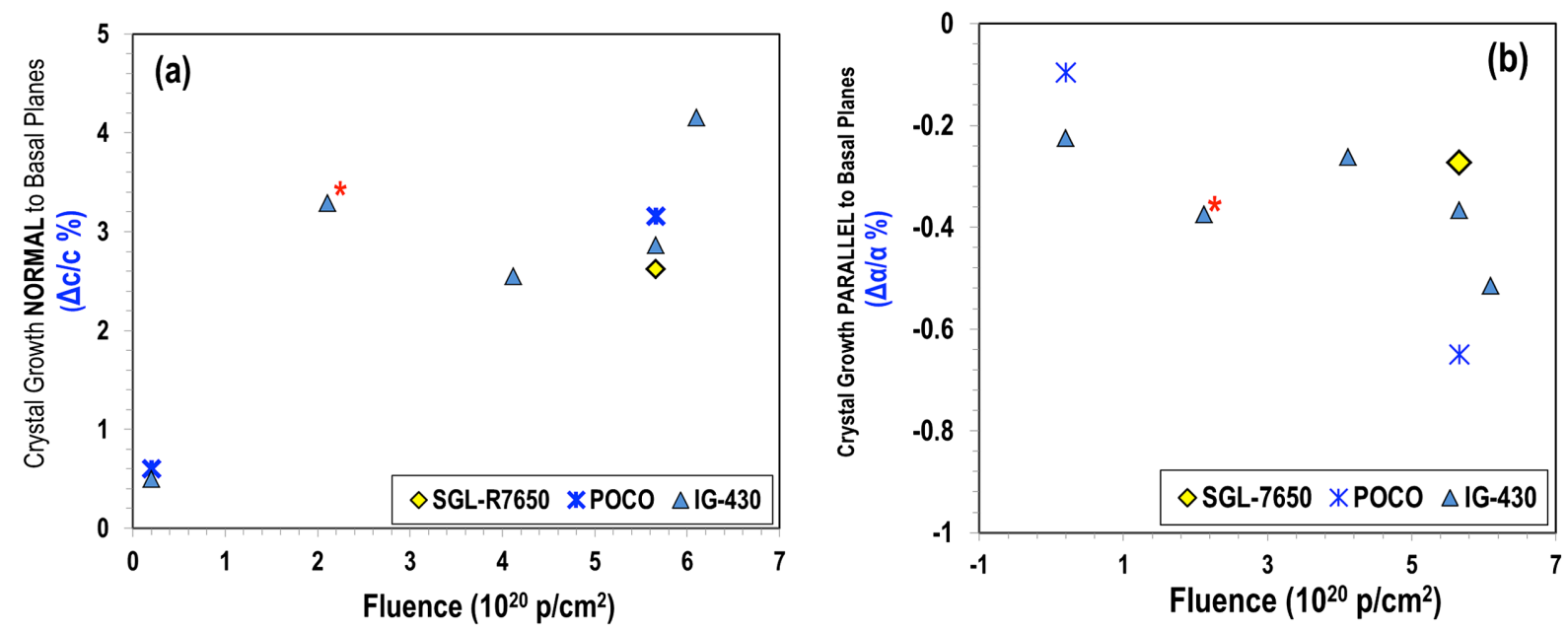

FIG. 19. Dimensional changes in $c$ and $a$ axes of proton-irradiated graphite grades POCO, IG 430 and SGL to fluence up to $6.1 \times 10^{20} \mathrm{p} / \mathrm{cm}^{2}$. Asterisks indicate data for irradiation temperature $\sim 150{ }^{\circ} \mathrm{C}$. 

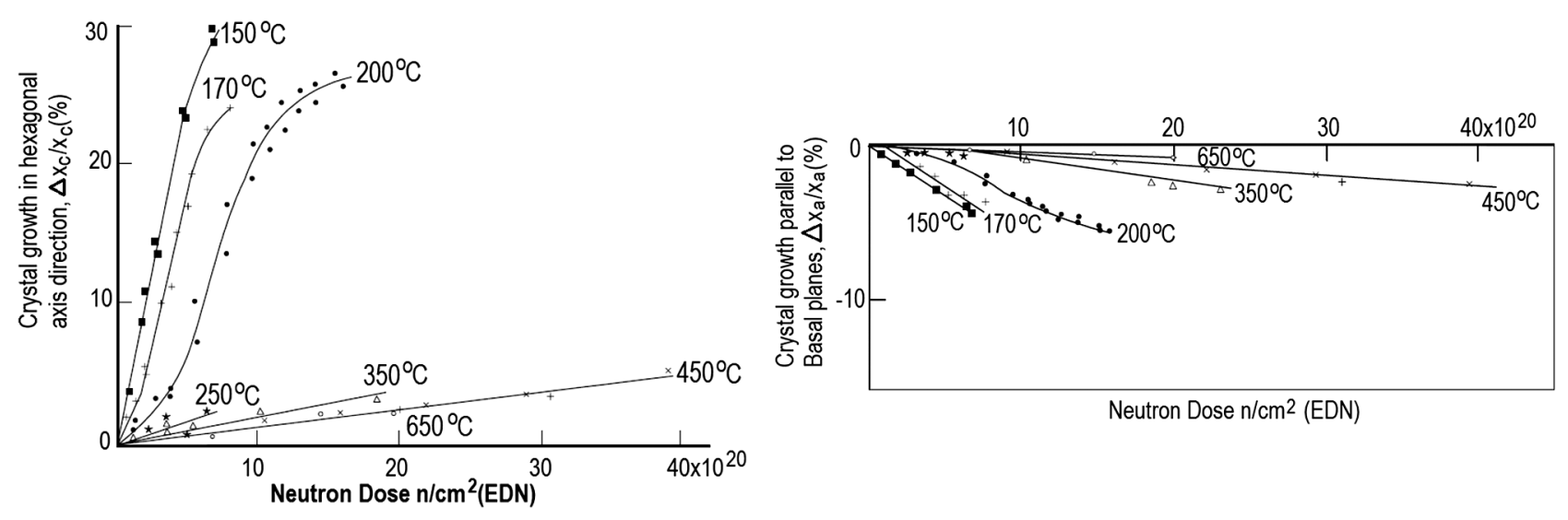

FIG. 20. Dimensional changes in $c$ and $a$ axes of pyrolytic graphite by energetic (>100 eV) neutrons (after Marsden [11]).

at least for the irradiation temperature of interest of the present study $\left(<200^{\circ} \mathrm{C}\right)$, provides for an excellent opportunity to utilize available neutron-based damage data $[8-10,12,13,20]$ for proton-based studies. To further scrutinize this proton-neutron damage correlation the evolution of the (002) diffraction peak characterizing the graphite lattice normal to the basal planes was studied in detail.

Shown in Fig. 21(a) is the evolution of the (002) diffraction peak with fluence produced in this study. It includes the unirradiated graphite diffraction peak, the diffraction peak at a fluence just below the threshold $\left(3.24 \times 10^{20} \mathrm{p} / \mathrm{cm}^{2}\right)$ and the $(002)$ peaks above the threshold $\left(5.66 \times 10^{20} \mathrm{p} / \mathrm{cm}^{2}\right)$ for the three graphite grades, POCO, IG 430 and SGL. The decrease in peak height and the asymmetric broadening indicates distortion and strain of the lattice. It is apparent that a dramatic change occurs in the lattice of the proton-irradiated graphite across the threshold fluence of $\sim 5 \times 10^{20} \mathrm{p} / \mathrm{cm}^{2}$. It is important to point out the similarity between proton damage and fast neutron damage of graphite and in particular in the threshold fluence where broadening of the (002) diffraction peaks starts [Fig. 21(b)]. These correlation results, as deduced by this research work, provide further evidence that the effects of either protons or neutrons on graphite are quite similar and therefore use of available neutron data at higher fluences and perhaps different irradiation temperature regimes is possible.

\section{SUMMARY AND DISCUSSION}

Four graphite grades considered for pion production targets in the Long Baseline Neutrino Facility were irradiated to a peak fluence of $6.1 \times 10^{20} \mathrm{~cm}^{-2}$ of $181 \mathrm{MeV}$ protons from the Brookhaven Linac at the BLIP target station. Irradiation temperatures were estimated in the range of $120-200^{\circ} \mathrm{C}$. One of the objectives of the multifaceted postirradiation study was to assess irradiation-induced damage in POCO ZXF-5Q, Toyo Tanso IG 430, SGL R7650 and Carbone-Lorraine C-2020 graphite grades at these fluence levels, damage seen in terms of changes in physical and mechanical properties that include CTE, strength and Young's modulus change. Assessment and comparison at these fluence levels will provide a basis for either selecting or excluding certain graphite grades from further consideration.
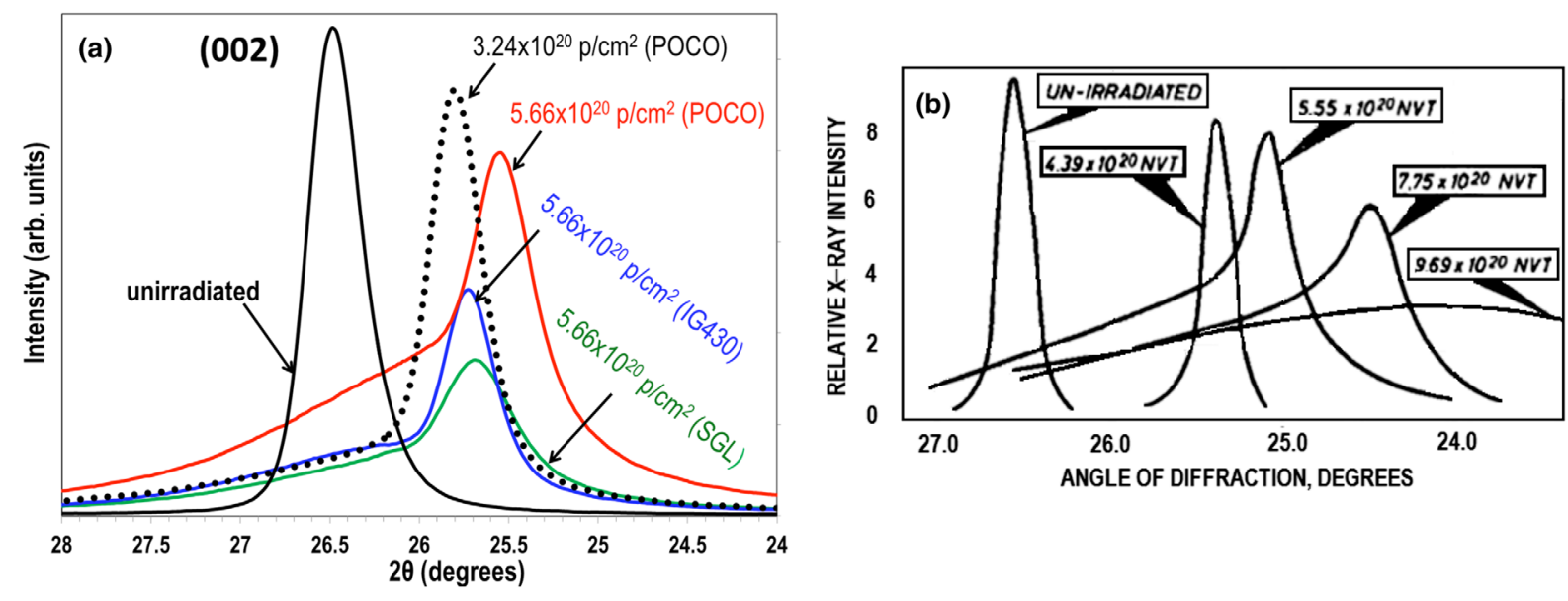

FIG. 21. (a) Evolution of (002) diffraction peak of graphite with proton fluence produced by the current study and (b) evolution of the (002) peak under fast neutrons (after Bollmann [9]). 
Evaluation of the four grades in the as-received condition revealed that the POCO grade exhibits the highest strength as well as the highest CTE. C-2020 appears to have the lowest CTE but also the lowest strength. C-2020 exhibits the least ordered structure between the four grades, a possible source of the wide scattering of its unirradiated strength data obtained during tension tests. X-ray diffraction of the POCO, IG 430 and SGL revealed that IG 430 has the lowest d-spacing and POCO the largest, an indication that IG 430 achieved higher graphitization in the manufacturing process.

Comparison of the four grades after exposure to the same proton fluence and irradiation temperature revealed the following.

There is an increase in CTE in all grades due to irradiation and it appears to reach a maximum within the range of $4.07-6.01 \times 10^{20} \mathrm{p} / \mathrm{cm}^{2}$. Similar observations were made for neutron irradiation of graphite for the same temperature regime. In these neutron damage studies [11] for fluences $>5 \times 10^{20} \mathrm{n} / \mathrm{cm}^{2}$ at room temperature and $5 \times 10^{20} \mathrm{n} / \mathrm{cm}^{2}$ at $225^{\circ} \mathrm{C}$ the CTE was observed to experience a reduction.

The mechanical strength of all four graphite grades, as observed in this study, increases with proton irradiation by varying factors. Highest percent increase is observed for C-2020 graphite ( factor of 3 ) followed by IG 430 ( $\sim$ factor of 2$)$, POCO graphite $(\sim 80 \%)$ and lastly SGL $(\sim 50 \%)$.

POCO, IG 430 and SGL appear to reach a maximum strength at approximately the same proton fluence $\left(\sim 3 \times 10^{20} \mathrm{p} / \mathrm{cm}^{2}\right)$ beyond which their strength continued to decrease. C-2020 reached its maximum strength at lower fluence $\left(\sim 10^{20} \mathrm{p} / \mathrm{cm}^{2}\right)$.

All four irradiated graphite grades appear to have experienced irradiation-induced creep even though the temperatures at which irradiation took place is relatively low $\left(\leq 200^{\circ} \mathrm{C}\right)$. This led to the significant increase of the Young's modulus ( $140 \%$ for IG 430$)$. Postirradiation annealing above the activation energy of graphite and the irradiation temperature recovered only a portion of the increase in Young's modulus.

Based on the above macroscopic observations of the proton-induced effects on the tested graphite grades it is assessed that establishing the existence of proton fluence thresholds for the increase of CTE, strength and Young's modulus is of significance. Two important aspects enhance the findings: (a) the threshold fluence is lower than the maximum obtained during proton irradiation and (b) these findings are in excellent agreement with neutron irradiation effects under similar fluences and irradiation temperatures.

X-ray diffraction of irradiated POCO, IG 430 and SGL graphite was conducted using high energy $\mathrm{x}$ rays at the NSLS synchrotron to explore radiation-induced changes in the lattice structure and lattice parameters. The $\mathrm{x}$-ray analysis revealed the following.
Increase in the d-spacing along the $c$-axis is observed for the three grades in the order of a few percent. POCO graphite experienced the higher percent increase in the c-parameter and showed clear tendency to increase its crystallinity accompanied by a turbostratic state. For all three grades asymmetric broadening of the (002) diffraction peak was observed for fluences $>5 \times 10^{20} \mathrm{p} / \mathrm{cm}^{2}$ indicating strong distortion of the lattice and strain.

The percent increase in $\mathrm{d}_{002}$-spacing, or $\mathrm{c}_{\mathrm{o}}$ parameter, observed for the three graphite grades due to proton irradiation agrees well with neutron irradiation effects on the same parameter and for the same temperature regime. This reinforces the findings from the macroscopic studies reported in this study where excellent agreement between effects of neutrons and protons was revealed.

Closer analysis of the evolution of the (002) diffraction peak with fluence revealed that there exists a dramatic shift and shape change of the peak around the fluence of $5 \times 10^{20} \mathrm{p} / \mathrm{cm}^{2}$. This tends to explain macroscopically observed evolution of physical and mechanical properties around the same fluence.

Most striking, however, is the similarity of the (002) peak evolution with the evolution of the same diffraction peak under neutron irradiation, a direct correlation reported for the first time in this study.

Based on both the macroscopic and microscopic assessment of the similarity in neutron and proton irradiation effects on graphite revealed in this study, the wealth of neutronrelated data accumulated over decades of research on graphite and which extend to much higher fluences than those achieved under proton irradiation as well as different temperature regimes can be utilized in proton-related studies.

The graphite grades of POCO, IG 430 and SGL, while exhibiting some variation of properties under irradiation, all adhere to the trends observed for graphite under neutron irradiation and therefore can remain under consideration for LBNF muon production targets. Establishing lifetimes for these three grades, while challenging, is now possible by relying on available neutron data on graphite based on anticipated irradiation temperature regimes. The study to date has established fluence thresholds for turn-around values of CTE, strength and Young's modulus, thresholds that were within the achieved fluence range. Lifetime parameters established for neutron effects on graphite, such as the fluence at which the graphite material volumetrically returns to the initial value can be utilized for guidance.

Future experiments which will explore different temperature regimes under proton irradiation and compare with the observation made under neutron irradiation are envisioned.

[1] M. Bogomilov et al., Neutrino factory, Phys. Rev. ST Accel. Beams 17, 121002 (2014).

[2] N. Simos et al., Long baseline neutrino experiment (LBNE) target material radiation damage from energetic 
protons of the Brookhaven Linear Isotope Production (BLIP) Facility, Report No. BNL-111826-2016-IR.

[3] A. Burkholtz, Irradiation damage in graphite, report prepared at the CEN, EUR 3056.e, 1966.

[4] D. G. Schweitzer, Activation energy for single interstitials in neutron-irradiated graphite and the absolute rate of formation of displaced atoms, Phys. Rev. 128, 556 (1962).

[5] J. Gittus, Creep, Viscoelasticity and Creep Fracture in Solids (Wiley, New York, 1975).

[6] N. Maruyama and M. Harayama, Neutron irradiation effect of thermal conductivity and dimensional change of graphite materials, J. Nucl. Mater. 195, 44 (1992).

[7] J.-P. Bonal, A. Kohyama, J. van der Laan, and L. L. Snead, Graphite, ceramics, and ceramic composites for high-temperature nuclear power systems, MRS Bull. 34, 28 (2009).

[8] W. T. Eeles, Diffuse diffraction phenomena from neutronirradiated graphite single crystals, Acta Cryst. A 24, 688 (1968).

[9] W. Bollmann, Electron-microscopic observations on radiation damage in graphite, Philos. Mag. 5, 621 (1960).

[10] B. T. Kelly, On the amorphization of graphite under neutron irradiation, J. Nucl. Mater. 172, 237 (1990).

[11] B. J. Marsden, Irradiation damage in graphite due to fast neutrons in fission and fusion systems, Report No. IAEA-TECDOC-1154.

[12] B. T. Kelly, The behavior of graphite under neutron irradiation, J. Vac. Sci. Technol. A 4, 1171 (1986).

[13] B. T. Kelly and J. E. Brocklehurst, Carbon 9, 783 (1971).
[14] L. L. Snead, T. D. Burchell, and Y. Katoh, Swelling of nuclear graphite and high quality carbon fiber composite under very high irradiation temperature, J. Nucl. Mater. 381, 55 (2008).

[15] T. Shibata et al., Oxidation damage evaluation by nondestructive method for graphite compounds in high temperature gas-cooled reactor, J. Solid Mech. Mater. Eng. 2, 166 (2008).

[16] N. V. Mokhov, The MARS Code System User's Guide, Report No. Fermilab-FN-628; N. V. Mokhov and S. I. Striganov, MARS15 overview, in Proceedings of the Hadronic Shower Simulation Workshop, Fermilab, 2006 AIP Conf. Proc. 896, 50 (2007); N. V. Mokhov et al., Prog. Nucl. Sci. Technol. 4, 496 (2014); http://dx.doi.org/10 .15669/pnst.4.496; http://www-ap.fnal.gov/MARS/.

[17] T. T. Böhlen, F. Cerutti, M. P. W. Chin, A. Fassò, A. Ferrari, P. G. Ortega, A. Mairani, P. R. Sala, G. Smirnov, and V. Vlachoudis, The FLUKA code: Developments, and challenges for high energy, and medical applications, Nucl. Data Sheets 120, 211 (2014).

[18] A. Ferrari, P. R. Sala, A. Fasso, and J. Ranft, FLUKA: A multiparticle transport code, Reports No. CERN-2005-10, No. INFN/TC_05/11, and No. SLAC-R-773.

[19] LS-DYNA, Keyword User's Manual, Version 971, Livermore Software Technology Corporation, 2007.

[20] W. T. Eeles, Interpretation of the diffuse diffraction phenomena of neutron-irradiated graphite crystals, Philos. Mag. 32, 1273 (1975). 